\title{
Synthesis and Semiconducting Characteristics of the BF2 Complexes of Bisbenzothiophene Fused Azadipyrromethenes
}

Wanle Sheng, ${ }^{\dagger} \S$ Fei Chang ${ }^{\dagger}$, Qinghua $\mathrm{Wu},{ }^{\dagger}$ Erhong Hao, ${ }^{\dagger}$ Lijuan Jiao*†, Jie-Yu Wang** and Jian Pei*:

${ }^{\dagger}$ Laboratory of Functional Molecular Solids, Ministry of Education; School of Chemistry and Materials Science, Anhui Normal University, Wuhu, 241002, China. ${ }^{\ddagger}$ Beijing National Laboratory for Molecular Sciences, College of Chemistry, Peking University, 100871, China.

$\S^{\S}$ Department of Chemistry, BengBu Medical College, Bengbu 233030, China.

*E-mail: jiao421@ahnu.edu.cn; jieyuwang@pku.edu.cn; jianpei@pku.edu.cn.

\section{Contents:}

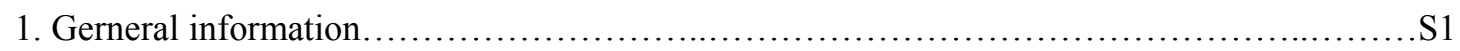

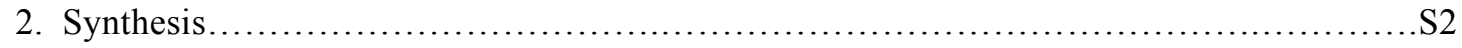

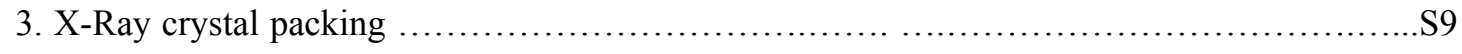

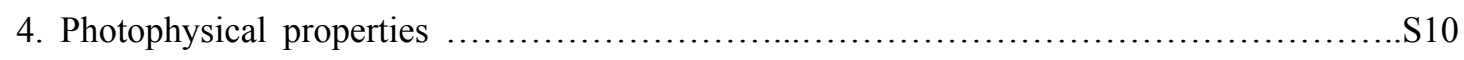

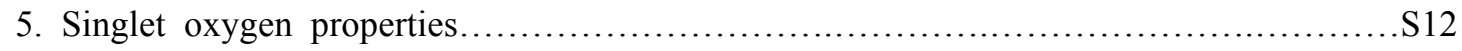

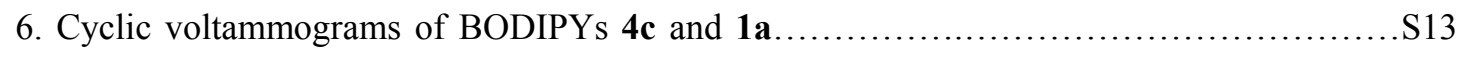

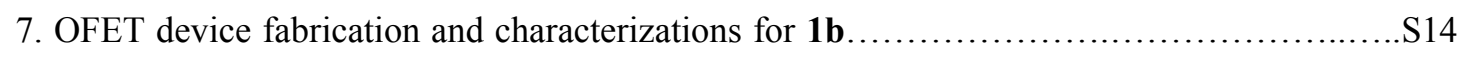

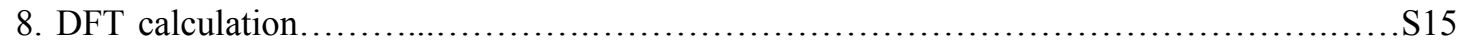

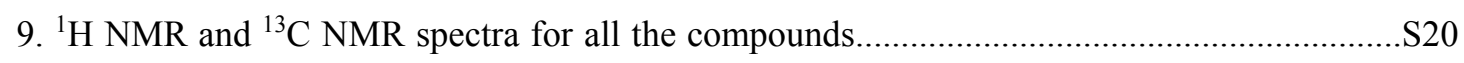

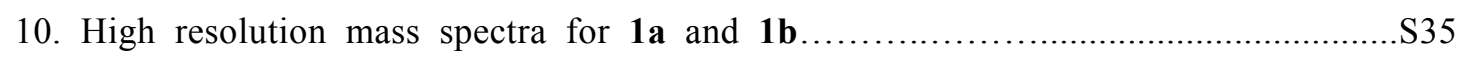

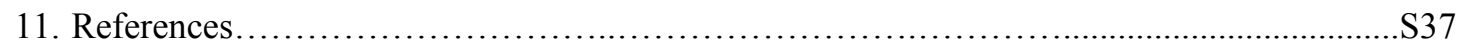




\section{Gerneral information}

Reagents and solvents were used as received from commercial suppliers unless noted otherwise. All reactions were performed in oven-dried glassware unless otherwise stated, and were monitored by TLC using $0.25 \mathrm{~mm}$ silica gel plates with UV indicator (60F-254). An oil bath with temperature control was used for all reactions that require heating. Nuclear Magnetic Resonance (NMR) spectra were recorded on $300 \mathrm{MHz}$ or $500 \mathrm{MHz}$ Bruker AVANCE III spectrometers. Chemical shifts are reported in ppm. Coupling constants ( $J$ values) are reported in Hertz. ${ }^{1} \mathrm{H}$ NMR chemical shifts were referenced to TMS (0 ppm). ${ }^{13} \mathrm{C}$ NMR chemical shifts were referenced to $\mathrm{CDCl}_{3}$ (77.00 ppm). High-resolution mass spectra (HRMS) were obtained using APCI-TOF in positive mode.

UV-visible absorption spectra and fluorescence emission spectra were recorded on commercial spectrophotometers at room temperature. Relative fluorescence quantum efficiencies of dyes were obtained by comparing the areas under the corrected emission spectrum of the test sample in various solvents with reference compound. Fluorescence quantum yields were calculated using 1,7-diphenyl-3,5-di(4-methoxyphenyl)azaBODIPY ( $\phi=0.36$ in chloroform) as reference. ${ }^{1}$ Non-degassed, spectroscopic grade solvents and a $10 \mathrm{~mm}$ quartz cuvette were used. Dilute solutions $(0.01<\mathrm{A}<$ 0.05 ) were used to minimize the reabsorption effects. Quantum yields were determined using the following equation: ${ }^{2}$

$$
\Phi_{x}=\Phi_{r} \times \frac{F_{x}}{F_{r}} \times \frac{1-10^{-A_{r}\left(\lambda_{\mathrm{ex}}\right)}}{1-10^{-A_{x}\left(\lambda_{\mathrm{ex}}\right)}} \times \frac{n_{x}^{2}}{n_{r}^{2}}
$$

The subscripts $\mathrm{x}$ and $\mathrm{r}$ refer respectively to our sample $\mathrm{x}$ and reference (standard) fluorophore $r$ with known quantum yield $\Phi_{r}$ in a specific solvent, $F$ stands for the spectrally corrected, integrated fluorescence spectra, A $\left(\lambda_{\text {ex }}\right)$ denotes the absorbance at the used excitation wavelength $\lambda_{\mathrm{ex}}$, and $n$ represents the refractive index of the solvent (in principle at the average emission wavelength).

Crystal of dye 1a (CCDC 1548795) suitable for X-ray analysis was obtained by slow diffusion of anhydrous ethanol into its dichloromethane solution (less than one week period) at room temperature. A suitable crystal was chosen and mounted on a glass fiber using grease. Data were collected using a diffractometer equipped with a graphite crystal monochromator situated in the incident beam for data collection at room temperature. Cell parameters were retrieved using SMART ${ }^{3}$ software and refined using $\mathrm{SAINT}^{4}$ on all observed reflections. The determination of unit cell parameters and data collections were performed with Mo K $\alpha$ radiation $(\lambda)$ at $0.71073 \AA$. Data reduction was performed using the SAINT software, which corrects for Lp and decay. The structure was solved by the direct method using the SHELXS-974 program and refined by least squares method on F, 1 SHELXL-97, ${ }^{5}$ incorporated in SHELXTL V5.10. ${ }^{6}$ 


\section{Synthesis}<smiles>[R]c1ccc(C=O)cc1</smiles><smiles>[R]c1ccc(/C=C/C(=O)c2ccccc2Br)cc1</smiles><smiles>CCO[N+](=O)[O-]</smiles>

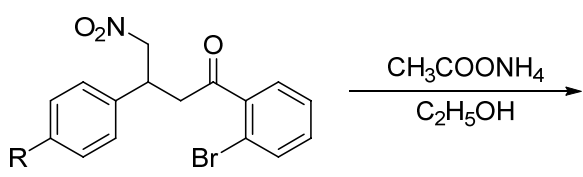<smiles>[R]c1ccc(C2=NC(c3ccccc3Br)=C/C2=N\c2[nH]c(-c3ccccc3Br)cc2-c2ccc([R])cc2)cc1</smiles>

$$
\begin{array}{ll}
\mathbf{5} \mathbf{a} \mathrm{R}=t-\mathrm{Bu} & 81 \% \\
\mathbf{5 b R}=\mathrm{OC}_{12} \mathrm{H}_{25} & 90 \%
\end{array}
$$

Scheme S1. Synthesis of compounds $\mathbf{3 a}$ and $\mathbf{3 b}$.

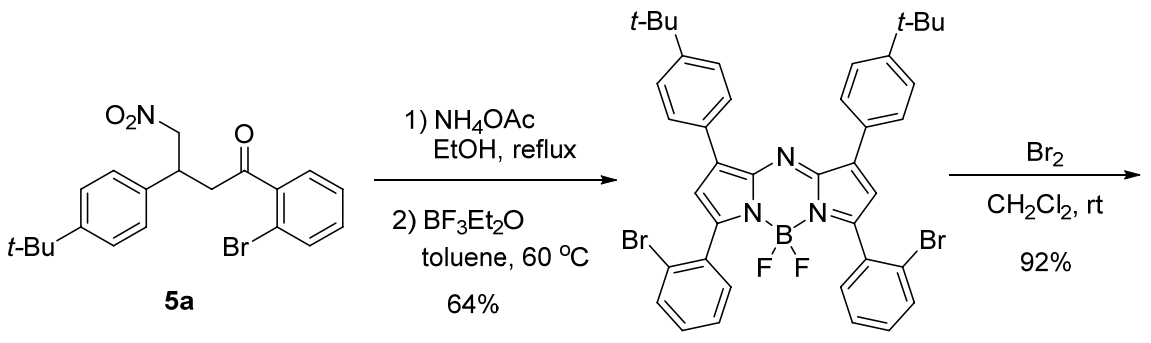

$4 a$

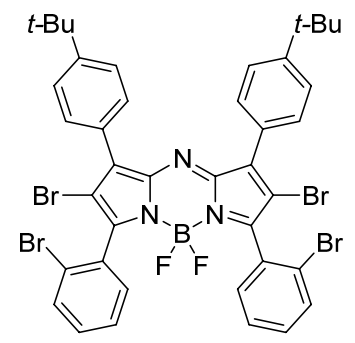

4b

Scheme S2. Synthesis of AzaBODIPYs 4a and $\mathbf{4 b .}$<smiles>CC(=O)c1ccc(C(C)O)cc1</smiles><smiles>CC(C)(C)c1ccc(/C=C/C(=O)c2ccccc2)cc1</smiles><smiles>CCO[N+](C)(C)OCCO</smiles>
$6 c$<smiles>CC(C)(C)c1ccc(C(CC(=O)c2ccccc2)C[N+](=O)[O-])cc1</smiles>

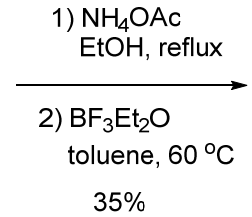<smiles></smiles>

Scheme S3. Synthesis of reference compound AzaBODIPY 4c. 


\section{Synthesis of compounds 6a-c:}

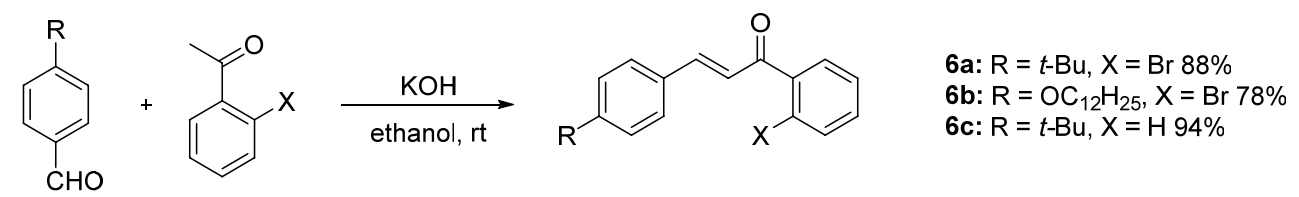

4-tert-butylbenzaldehyde (3.24 g, $20.0 \mathrm{mmol})$ and o-bromoacetophnone (3.96 g, 20.0 $\mathrm{mmol})$ was dissolved in anhydrous ethanol $(30 \mathrm{~mL})$, and $\mathrm{KOH}(2.20 \mathrm{~g})$ was added. The mixture was stirred at room temperature for $10 \mathrm{~min}$, then the reaction mixture was poured into water, extracted with dichloromethane. The organic layer was evaporated to dryness under reduced pressure to give compound 6a as a light yellow oil (6.02 g, 88 \%). ${ }^{1} \mathrm{H}$ NMR (300 MHz, $\left.\mathrm{CDCl}_{3}, \mathrm{ppm}\right) \delta 7.64(\mathrm{~d}, J=7.8 \mathrm{~Hz}, 1 \mathrm{H}), 7.50(\mathrm{~d}, J=8.4$ $\mathrm{Hz}, 2 \mathrm{H}), 7.43-7.29(\mathrm{~m}, 6 \mathrm{H}), 7.06$ (d, $J=15.9 \mathrm{~Hz}, 1 \mathrm{H}), 1.32$ (s, 9H). ${ }^{13} \mathrm{C}$ NMR (125 $\left.\mathrm{MHz}, \mathrm{CDCl}_{3}, \mathrm{ppm}\right) \delta 194.8,154.6,146.7,141.2,133.4,131.6,131.2,129.1,128.5$, 127.3, 126.0, 125.4, 119.4, 34.9, 31.1. HRMS (APCI) Calcd. for $\mathrm{C}_{19} \mathrm{H}_{20} \mathrm{BrO}[\mathrm{M}+\mathrm{H}]^{+}$: 343.0698 , found 343.0692 .

6b was prepared in 78\% yield (7.18 g) from 4-dodecyloxybenzaldehyde (5.60 g, 20.0 mmol) and o-bromoacetophnones (3.96 g, $20.0 \mathrm{mmol})$ using the above procedure. ${ }^{1} \mathrm{H}$ NMR (500 MHz, $\left.\mathrm{CDCl}_{3}, \mathrm{ppm}\right) \delta 7.63(\mathrm{~d}, J=8.0 \mathrm{~Hz}, 1 \mathrm{H}), 7.49$ (d, $\left.J=9.0 \mathrm{~Hz}, 2 \mathrm{H}\right)$, $7.41-7.29(\mathrm{~m}, 4 \mathrm{H}), 6.95(\mathrm{~d}, J=16.0 \mathrm{~Hz}, 1 \mathrm{H}), 6.89$ (d, $J=9.0 \mathrm{~Hz}, 2 \mathrm{H}), 3.98$ (t, $J=6.5$ $\mathrm{Hz}, 2 \mathrm{H}), 1.81-1.75(\mathrm{~m}, 2 \mathrm{H}), 1.47-1.42(\mathrm{~m}, 2 \mathrm{H}), 1.36-1.26(\mathrm{~m}, 16 \mathrm{H}), 0.88(\mathrm{t}, J=7.0$ $\mathrm{Hz}, 3 \mathrm{H}) .{ }^{13} \mathrm{C} \mathrm{NMR}\left(125 \mathrm{MHz}, \mathrm{CDCl}_{3}\right.$, ppm) $\delta 194.7,161.6,146.8,141.4,133.3,131.0$, 130.4, 129.0, 127.2, 126.8, 123.4, 119.4, 114.9, 68.2, 31.9, 29.6(9), 29.6(7), 29.5(2), 29.5(0), 29.3, 29.1, 25.9, 22.6, 14.1. HRMS (APCI) calcd. for $\mathrm{C}_{27} \mathrm{H}_{36} \mathrm{BrO}_{2}[\mathrm{M}+\mathrm{H}]^{+}$: 471.1899 , found 471.1887 .

6c was prepared in 94\% yield (3.97 g) from 4-tert-butylbenzaldehyde (2.59 g, 16.0 $\mathrm{mmol})$ and acetophnone $(1.92 \mathrm{~g}, 16.0 \mathrm{mmol})$ using the above procedure. ${ }^{1} \mathrm{H}$ NMR (300 $\left.\mathrm{MHz}, \mathrm{CDCl}_{3}, \mathrm{ppm}\right) \delta 8.02(\mathrm{~d}, J=7.2 \mathrm{~Hz}, 2 \mathrm{H}), 7.81(\mathrm{~d}, J=15.6 \mathrm{~Hz}, 1 \mathrm{H}), 7.61-7.43$ (m, 8H), $1.34(\mathrm{~s}, 9 \mathrm{H}) .{ }^{13} \mathrm{C} \mathrm{NMR}\left(75 \mathrm{MHz}, \mathrm{CDCl}_{3}\right.$, ppm) $\delta 190.5,154.1,144.8,138.3$, 132.6, 132.0, 128.6, 128.4, 128.3, 125.9, 121.2, 34.8, 31.1. HRMS (APCI) calcd. for $\mathrm{C}_{19} \mathrm{H}_{21} \mathrm{O}[\mathrm{M}+\mathrm{H}]^{+}: 265.1587$, found 265.1580 . 
Synthesis of compounds 5a-c:
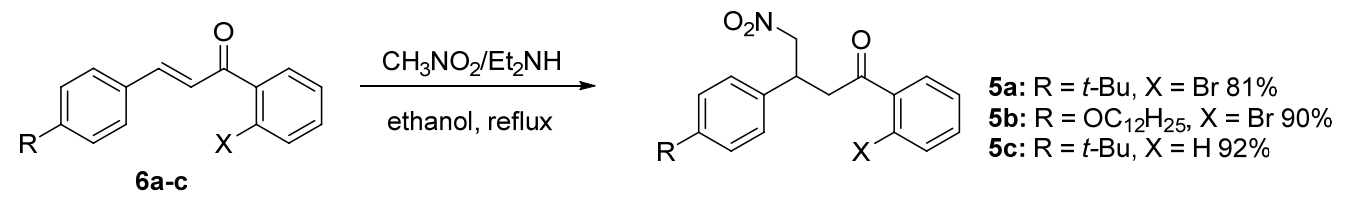

Compound 6a $(5.13 \mathrm{~g}, 15.0 \mathrm{mmol})$ in anhydrous ethanol $(40 \mathrm{~mL})$ was treated with diethylamine $(8 \mathrm{~mL})$ and nitromethane $(8 \mathrm{~mL})$. The solution was stirred for $24 \mathrm{~h}$ under reflux and was then concentrated under vacuum. The residue was poured into water, extracted with dichloromethane to afford the oiled compound $5 \mathbf{a}(4.89 \mathrm{~g}, 81 \%) .{ }^{1} \mathrm{H}$ NMR (300 MHz, $\mathrm{CDCl}_{3}$, ppm) $\delta 7.56$ (d, $\left.J=6.9 \mathrm{~Hz}, 1 \mathrm{H}\right), 7.54$ - 7.24 (m, 4H), 7.21 $7.14(\mathrm{~m}, 3 \mathrm{H}), 4.83$ - $4.65(\mathrm{~m}, 2 \mathrm{H}), 4.20$ - $4.13(\mathrm{~m}, 1 \mathrm{H}), 3.46-3.31(\mathrm{~m}, 2 \mathrm{H}), 1.28(\mathrm{~s}$, 9H). ${ }^{13} \mathrm{C}$ NMR (125 MHz, $\left.\mathrm{CDCl}_{3}, \mathrm{ppm}\right) \delta 201.1,150.7,140.9,135.3,133.6,131.8$, 128.4, 127.4, 127.1, 125.8, 118.4, 79.3, 45.6, 39.0, 34.4, 31.2. HRMS (APCI) Calcd. for $\mathrm{C}_{20} \mathrm{H}_{23} \mathrm{O}_{3} \mathrm{NBr}[\mathrm{M}+\mathrm{H}]^{+}$404.0861, found 404.0857.

5b was prepared in 90\% yield $(7.17 \mathrm{~g})$ from $6 \mathbf{b}(7.05 \mathrm{~g}, 15.0 \mathrm{mmol})$ using the above procedure. ${ }^{1} \mathrm{H}$ NMR $\left(500 \mathrm{MHz}, \mathrm{CDCl}_{3}, \mathrm{ppm}\right) \delta 7.56(\mathrm{~d}, J=8.0 \mathrm{~Hz}, 1 \mathrm{H}), 7.33-7.26(\mathrm{~m}$, 2H), $7.20(\mathrm{~d}, J=7.0 \mathrm{~Hz}, 1 \mathrm{H}), 7.13(\mathrm{~d}, J=8.5 \mathrm{~Hz}, 2 \mathrm{H}), 6.83$ (d, $J=8.5 \mathrm{~Hz}, 2 \mathrm{H}), 4.78$ $4.62(\mathrm{~m}, 2 \mathrm{H}), 4.13$ - $4.08(\mathrm{~m}, 1 \mathrm{H}), 3.43-3.32(\mathrm{~m}, 2 \mathrm{H}), 1.77$ - $1.74(\mathrm{~m}, 2 \mathrm{H}), 1.43-1.34$ $(\mathrm{m}, 2 \mathrm{H}), 1.33-1.26(\mathrm{~m}, 16 \mathrm{H}), 0.88$ (t, $J=7.0 \mathrm{~Hz}, 3 \mathrm{H}) .{ }^{13} \mathrm{C} \mathrm{NMR}\left(125 \mathrm{MHz}, \mathrm{CDCl}_{3}\right.$, ppm) $\delta 201.1,158.7,141.0,133.6,131.8,130.1,128.5,128.4,127.5,118.5,114.9$, 79.6, 70.0, 45.7, 38.8, 31.9, 29.6(1), 29.6(9), 29.6(5), 29.5, 29.4, 29.3, 29.2 , 26.0, 22.6, 14.1. HRMS (APCI) calcd. for $\mathrm{C}_{28} \mathrm{H}_{39} \mathrm{BrNO}_{4}[\mathrm{M}+\mathrm{H}]^{+}:$532.2062, found 532.2049.

5c was prepared in 92\% yield (4.49 $\mathrm{g}$ ) from $6 \mathrm{c}$ (3.96 g, $15.0 \mathrm{mmol})$ using the above procedure. ${ }^{1} \mathrm{H}$ NMR $\left(300 \mathrm{MHz}, \mathrm{CDCl}_{3}, \mathrm{ppm}\right) \delta 7.92(\mathrm{~d}, J=7.8 \mathrm{~Hz}, 2 \mathrm{H}), 7.60$ - 7.55 (m, 1H), $7.48-7.43(\mathrm{~m}, 2 \mathrm{H}), 7.34$ (d, $J=8.4 \mathrm{~Hz}, 2 \mathrm{H}), 7.21$ (d, $J=8.1 \mathrm{~Hz}, 2 \mathrm{H}), 4.86-4.65$ $(\mathrm{m}, 2 \mathrm{H}), 4.26-4.16(\mathrm{~m}, 1 \mathrm{H}), 3.53-3.37(\mathrm{~m}, 2 \mathrm{H}), 1.29(\mathrm{~s}, 9 \mathrm{H}) .{ }^{13} \mathrm{C}$ NMR $(75 \mathrm{MHz}$, $\left.\mathrm{CDCl}_{3}, \mathrm{ppm}\right) \delta 197.0,150.6,136.3,136.0,133.5,128.7,128.0,127.0,125.9,79.5,41.6$, 38.7, 34.4, 31.2. HRMS (APCI) calcd. for $\mathrm{C}_{20} \mathrm{H}_{24} \mathrm{NO}_{3}[\mathrm{M}+\mathrm{H}]^{+}: 326.1751$, found 326.1732 . 


\section{Synthesis of azaBODIPYs $4 a$ and $4 c$ following the literature: ${ }^{7}$}

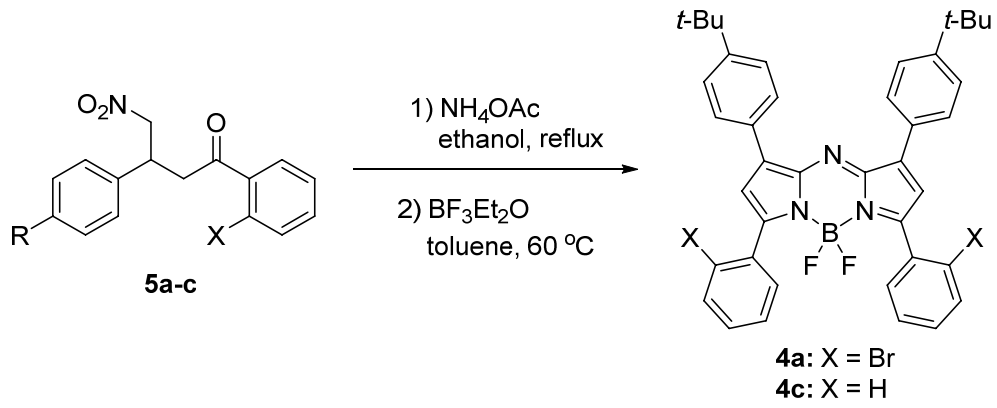

Compound 5a (4.03 g, $10.0 \mathrm{mmol})$ and ammonium acetate $(11.6 \mathrm{~g}, 150 \mathrm{mmol})$ in ethanol $(50 \mathrm{ml})$ were heated under reflux for $24 \mathrm{~h}$. The solution was then cooled to room temperature and the precipitate was filtered. The solid was washed with ethanol, dried under vacuum. Then it was dissolved in toluene. Triethylamine $(10 \mathrm{ml}, 72 \mathrm{mmol})$ and borontrifluoride diethyletherate $(15 \mathrm{ml}$, $0.14 \mathrm{~mol}$ ) were added, and the reaction mixture was stirred at $65^{\circ} \mathrm{C}$ for $2 \mathrm{~h}$. The reaction mixture was poured into water, extracted with dichloromethane, dried over anhydrous $\mathrm{Na}_{2} \mathrm{SO}_{4}$, filtered and evaporated under vacuum. The crude product was purified by column chromatograph (silica gel, hexane : dichloromethane $=7: 1)$ to give $4 \mathbf{a}$ as red powder (1.23 g, $64 \%)$. ${ }^{1} \mathrm{H}$ NMR (300 $\left.\mathrm{MHz}, \mathrm{CDCl}_{3}, \mathrm{ppm}\right) \delta 8.03(\mathrm{~d}, J=8.4 \mathrm{~Hz}, 4 \mathrm{H}), 7.72-7.64(\mathrm{~m}, 4 \mathrm{H}), 7.51(\mathrm{~d}, J=$ $8.4 \mathrm{~Hz}, 4 \mathrm{H}), 7.37$ (t, $J=7.4 \mathrm{~Hz}, 2 \mathrm{H}), 7.9-7.24(\mathrm{~m}, 4 \mathrm{H}), 6.90(\mathrm{~s}, 2 \mathrm{H}), 1.40$ (s, $18 \mathrm{H}) .{ }^{13} \mathrm{C} \mathrm{NMR}\left(125 \mathrm{MHz}, \mathrm{CDCl}_{3}, \mathrm{ppm}\right) \delta 158.4,153.2,145.0,143.9,133.0$, 132.9, 131.7, 131.1, 129.6, 129.3, 126.9, 125.7, 122.6, 120.0, 34.9, 31.2. HRMS (APCI) Calcd. For $\mathrm{C}_{40} \mathrm{H}_{37} \mathrm{~N}_{3} \mathrm{BBr}[81] \mathrm{BrF}_{2}[\mathrm{M}+\mathrm{H}]^{+}: 768.1395$ found 768.1390 .

4c was prepared in 35\% yield (1.07 $\mathrm{mg})$ from $5 \mathbf{c}(3.25 \mathrm{mg}, 10.0 \mathrm{mmol})$ using the same procedure for 4a. ${ }^{1} \mathrm{H}$ NMR (300 $\left.\mathrm{MHz}, \mathrm{CDCl}_{3}, \mathrm{ppm}\right) \delta 8.06-8.00(\mathrm{~m}, 8 \mathrm{H}), 7.51-7.47$ (m, 10H), 7.00 (s, 2H), 1.39 (s, 18H). $\left.{ }^{13} \mathrm{C} \mathrm{NMR} \mathrm{(75} \mathrm{MHz,} \mathrm{CDCl}_{3}, \mathrm{ppm}\right) \delta 159.4,152.9$, 145.7, 144.2, 131.7, 130.8, 129.6, 129.5, 129.2, 128.6, 125.6, 118.5, 34.8, 31.2. HRMS (APCI) calcd. for $\mathrm{C}_{40} \mathrm{H}_{39} \mathrm{BF}_{2} \mathrm{~N}_{3}[\mathrm{M}+\mathrm{H}]^{+}:$610.3205, found 610.3169 . 


\section{Synthesis of tetrabromoazaBODIPY 4b:}

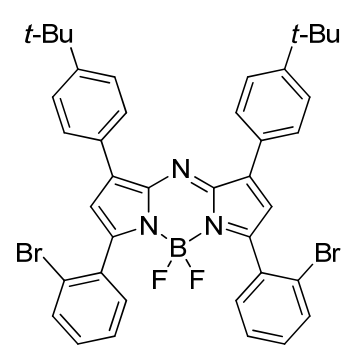

$4 a$

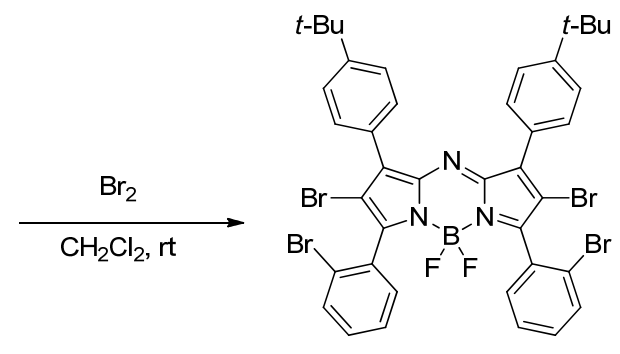

$4 b$

To $4 \mathbf{a}(765 \mathrm{mg}, 1.00 \mathrm{mmol})$ in $100 \mathrm{~mL}$ of dichloromethane was dropwisely added bromine (320 mg, $2.00 \mathrm{mmol}$ ) in $15 \mathrm{~mL}$ of dichloromethane. The reaction mixture was stirred at room temperature for 5 minutes, quenched by adding saturated aqueous solution of $\mathrm{Na}_{2} \mathrm{~S}_{2} \mathrm{O}_{3}$. Organic layer was collected, dried over anhydrous $\mathrm{Na}_{2} \mathrm{SO}_{4}$, and evaporated under vacuum. The crude product was purified by recrystallization from $\mathrm{CH}_{2} \mathrm{Cl}_{2} /$ methanol to give $4 \mathbf{b}$ in $89 \%$ yield (846 mg). ${ }^{1} \mathrm{H}$ NMR (300 $\mathrm{MHz}, \mathrm{CDCl}_{3}, \mathrm{ppm}$ ) $\delta 7.93(\mathrm{~d}, J=8.1 \mathrm{~Hz}, 4 \mathrm{H}), 7.64(\mathrm{~d}, J=7.8 \mathrm{~Hz}, 2 \mathrm{H}), 7.50(\mathrm{~d}, J=8.4 \mathrm{~Hz}, 4 \mathrm{H}), 7.45$ $7.29(\mathrm{~m}, 6 \mathrm{H}), 1.38$ (s, 18H). ${ }^{13} \mathrm{C}$ NMR (125 MHz, $\left.\mathrm{CDCl}_{3}, \mathrm{ppm}\right) \delta 158.1,153.3,144.2$, 142.2, 132.6, 131.6, 131.3, 131.2, 130.6, 127.7, 126.9, 125.1, 123.3, 110.2, 34.9, 31.2. HRMS (APCI) calcd. for $\mathrm{C}_{40} \mathrm{H}_{35} \mathrm{BBr}_{2}[81] \mathrm{Br}_{2} \mathrm{~F}_{2} \mathrm{~N}_{3} \quad[\mathrm{M}+\mathrm{H}]^{+}:$923.9605, found 923.9582.

\section{Synthesis of azadipyrromethenes $3 \mathrm{a}$ and $3 \mathrm{~b}$ :}
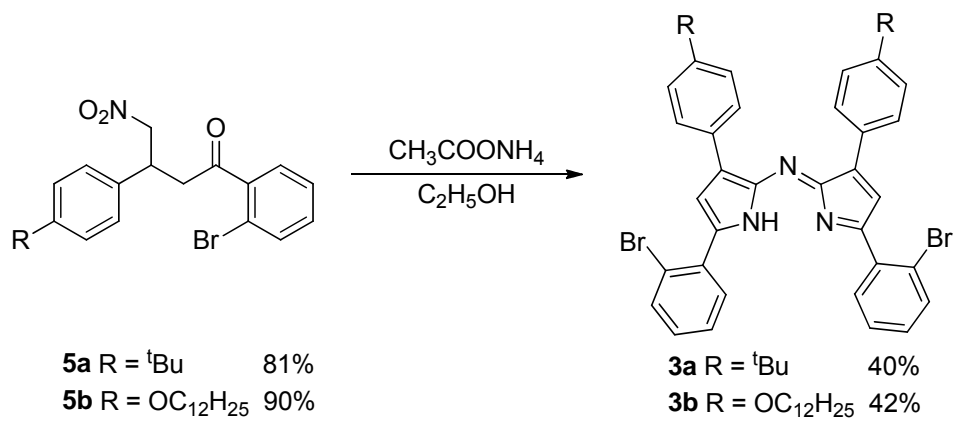

Compound 5a $(4.03 \mathrm{mg}, 10.0 \mathrm{mmol})$ and ammonium acetate $(11.6 \mathrm{~g}, 150 \mathrm{mmol})$ in ethanol $(50 \mathrm{~mL})$ were stirred for $24 \mathrm{~h}$ under reflux. The solution was then cooled to room temperature and the precipitate was filtered. The solid was washed with ethanol, dried under vacuum to give 3a (1.43 g, $40 \%$ ). ${ }^{1} \mathrm{H}$ NMR (300 $\left.\mathrm{MHz}, \mathrm{CDCl}_{3}, \mathrm{ppm}\right) \delta$ $7.96(\mathrm{~d}, J=6.3 \mathrm{~Hz}, 4 \mathrm{H}), 7.85(\mathrm{~s}, 2 \mathrm{H}), 7.70(\mathrm{~d}, J=7.8 \mathrm{~Hz}, 2 \mathrm{H}), 7.47-7.40(\mathrm{~m}$, $6 \mathrm{H}), 7.29$ - $7.24(\mathrm{~m}, 4 \mathrm{H}), 1.39$, (s, 18H). $\left.{ }^{13} \mathrm{C} \mathrm{NMR} \mathrm{(75} \mathrm{MHz,} \mathrm{CDCl}_{3}, \mathrm{ppm}\right) \delta 155.1$, 
$151.1,148.9,141.5,134.4,133.4,131.0,130.8,130.5,129.1,127.6,125.1,121.4$, 118.0, 34.7, 31.3. HRMS (APCI) Calcd. for $\mathrm{C}_{40} \mathrm{H}_{38} \mathrm{Br}[81] \mathrm{BrN}_{3}[\mathrm{M}+\mathrm{H}]^{+} 720.1412$, found 720.1385 .

3b was prepared in 42\% yield (2.04 g) from $\mathbf{5 b}(5.30 \mathrm{mg}, 10.0 \mathrm{mmol})$ and ammonium acetate $(11.6 \mathrm{~g}, 150 \mathrm{mmol})$ using the above procedure. ${ }^{1} \mathrm{H}$ NMR $\left(500 \mathrm{MHz}, \mathrm{CDCl}_{3}\right.$, ppm) $\delta 8.00(\mathrm{~d}, J=9.0 \mathrm{~Hz}, 4 \mathrm{H}), 7.83(\mathrm{~d}, J=7.0 \mathrm{~Hz}, 2 \mathrm{H}), 7.69(\mathrm{~d}, J=8.0 \mathrm{~Hz}, 2 \mathrm{H}), 7.40$ (t, $J=7.5 \mathrm{~Hz}, 2 \mathrm{H}), 7.24$ (d, $J=7.5 \mathrm{~Hz}, 2 \mathrm{H}), 7.15$ (s, 2H), 6.96 (d, $J=9.0 \mathrm{~Hz}, 4 \mathrm{H}), 4.03$ $(\mathrm{t}, J=6.5 \mathrm{~Hz}, 4 \mathrm{H}), 1.86-1.81(\mathrm{~m}, 4 \mathrm{H}), 1.52-1.47(\mathrm{~m}, 4 \mathrm{H}), 1.39-1.2(\mathrm{~m}, 32 \mathrm{H}), 0.88$ (t, $J=7.0 \mathrm{~Hz}, 6 \mathrm{H}) .{ }^{13} \mathrm{C} \mathrm{NMR}\left(125 \mathrm{MHz}, \mathrm{CDCl}_{3}, \mathrm{ppm}\right) \delta 159.3,154.9,148.8,141.2$, 134.4, 133.5, 131.0, 130.4, 127.6, 126.3, 121.4, 117.1, 114.4, 68.1, 31.9, 29.7, 29.6, 29.5, 29.4, 29.3, 26.1, 22.7, 14.1. HRMS (APCI) calcd. for $\mathrm{C}_{56} \mathrm{H}_{70} \mathrm{Br}[81] \mathrm{BrN}_{3} \mathrm{O}_{2}$ $[\mathrm{M}+\mathrm{H}]^{+}: 976.3809$, found 976.3810 .

\section{Synthesis of tetrabromoazadipyrromethenes $2 \mathrm{a}$ and $2 \mathrm{~b}$ :}

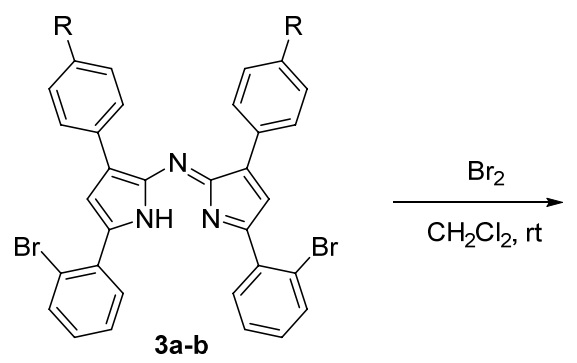

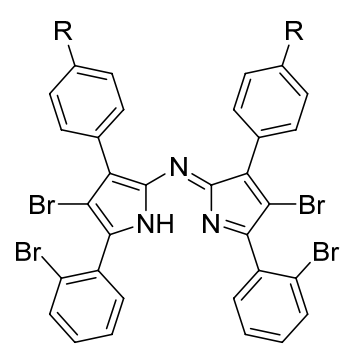

2a: $\mathrm{R}=t$-Bu

2b: $\mathrm{R}=\mathrm{OC}_{12} \mathrm{H}_{25}$

A solution of $\mathrm{Br}_{2}(320 \mathrm{mg}, 2.00 \mathrm{mmol})$ in dichloromethane $(5 \mathrm{~mL})$ was added dropwise into a solution of $3 \mathbf{a}(717 \mathrm{mg}, 1.00 \mathrm{mmol})$ in dichloromethane $(100 \mathrm{~mL})$ at room temperature. The reaction mixture was stirred at room temperature for 5 minutes, quenched by adding saturated aqueous solution of $\mathrm{Na}_{2} \mathrm{~S}_{2} \mathrm{O}_{3}$. Then it was washed with saturated sodium thiosulfate solution, the organic layer was dried over anhydrous $\mathrm{Na}_{2} \mathrm{SO}_{4}$, evaporated under vacuum. The crude product was purified by column chromatograph (silica gel, petroleum ether : dichloromethane $=3: 1)$ to give $2 \mathbf{a}(746$ mg, $85 \%$ ). ${ }^{1} \mathrm{H}$ NMR (300 MHz, $\left.\mathrm{CDCl}_{3}, \mathrm{ppm}\right) \delta 7.83(\mathrm{~d}, J=8.4 \mathrm{~Hz}, 4 \mathrm{H}), 7.70(\mathrm{~d}, J=$ $7.8 \mathrm{~Hz}, 2 \mathrm{H}), 7.61$ (d, $J=7.2 \mathrm{~Hz}, 2 \mathrm{H}), 7.45-7.42(\mathrm{~m}, 6 \mathrm{H}), 7.32$ (t, $J=7.6 \mathrm{~Hz}, 2 \mathrm{H})$, 1.38 (s, 18H). ${ }^{13} \mathrm{C}$ NMR (75 MHz, $\left.\mathrm{CDCl}_{3}, \mathrm{ppm}\right) \delta 154.2,151.3,147.5,139.4,133.4$, 133.0, 131.9, 131.0, 130.4, 128.8, 127.1, 124.7, 122.8, 108.8, 34.7, 31.3. HRMS (APCI) Calcd. for $\mathrm{C}_{40} \mathrm{H}_{36} \mathrm{Br}_{2}[81] \mathrm{Br}_{2} \mathrm{~N}_{3}[\mathrm{M}+\mathrm{H}]^{+}$: 877.9602, found 877.9582 . 
2b was prepared in $88 \%$ yield $(998 \mathrm{mg})$ from $\mathbf{3 b}(973 \mathrm{mg}, 1.00 \mathrm{mmol})$ and $\mathrm{Br}_{2}(320$ $\mathrm{mg}, 2.00 \mathrm{mmol})$ using the above procedure. ${ }^{1} \mathrm{H}$ NMR (500 $\left.\mathrm{MHz}, \mathrm{CDCl}_{3}, \mathrm{ppm}\right) \delta 7.83$ $(\mathrm{d}, J=8.8 \mathrm{~Hz}, 2 \mathrm{H}), 7.68(\mathrm{~d}, J=8.0 \mathrm{~Hz}, 1 \mathrm{H}), 7.57(\mathrm{~d}, J=7.5 \mathrm{~Hz}, 1 \mathrm{H}), 7.42$ (t, $J=7.5$ $\mathrm{Hz}, 1 \mathrm{H}), 7.31$ (t, $J=7.5 \mathrm{~Hz}, 1 \mathrm{H}), 6.93$ (d, $J=9.0 \mathrm{~Hz}, 2 \mathrm{H}), 4.02$ (t, $J=6.5 \mathrm{~Hz}, 2 \mathrm{H})$, $1.87-1.78(\mathrm{~m}, 2 \mathrm{H}), 1.52-1.44(\mathrm{~m}, 2 \mathrm{H}), 1.42-1.19(\mathrm{~m}, 16 \mathrm{H}), 0.89$ (t, $J=7.0 \mathrm{~Hz}, 3 \mathrm{H})$. ${ }^{13} \mathrm{C}$ NMR $\left(125 \mathrm{MHz}, \mathrm{CDCl}_{3}\right.$, ppm) $\delta 159.4,154.2,147.3,139.3,133.4,133.0,132.0$, 131.8, 130.9, 127.1, 124.1, 122.8, 113.8, 108.0, 68.1, 31.9, 29.7(0), 29.7(6), 29.5, 29.4, 29.3, 26.1, 22.7, 14.1. HRMS (APCI) calcd. for $\mathrm{C}_{56} \mathrm{H}_{68} \mathrm{Br}_{2}[81] \mathrm{Br}_{2} \mathrm{~N}_{3} \mathrm{O}_{2}[\mathrm{M}+\mathrm{H}]^{+}$: 1134.2004, found 1134.2017.

\section{Synthesis of dibenzothiophene fused azaBODIPYs 1a and 1b:}
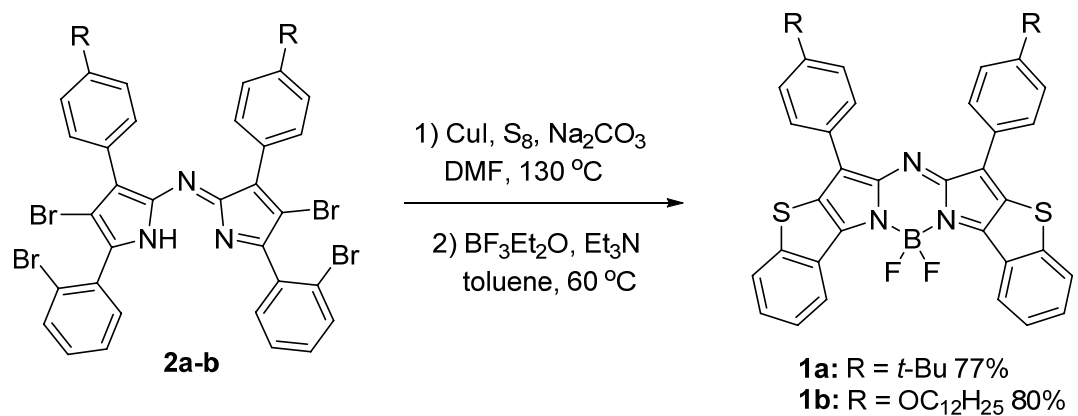

An oven-dried Schlenk tube was charged with 6a (100 mg, $0.115 \mathrm{mmol}), \mathrm{CuI}(8.8 \mathrm{mg}$, $0.046 \mathrm{mmol}$ ), sulfur powder (14.8 $\mathrm{mg}, 0.58 \mathrm{mmol}$ ) and $\mathrm{K}_{2} \mathrm{CO}_{3}(64 \mathrm{mg}, 0.463 \mathrm{mmol})$. The Schlenk tube was evacuated and backfilled with Ar, followed by the addition of DMF $(15 \mathrm{~mL})$. The reaction mixture was stirred at $130{ }^{\circ} \mathrm{C}$ for $15 \mathrm{~h}$. Upon cooling to room temperature, the reaction mixture was diluted with ethyl acetate $(5 \mathrm{~mL})$ and filtered through a pad of silica gel using additional ethyl acetate $(15 \mathrm{~mL})$ as the eluent. The filtrate was washed with water, dried over anhydrous $\mathrm{Na}_{2} \mathrm{SO}_{4}$, and concentrated under reduced pressure. Then the residue was dissolved in toluene $(20 \mathrm{~mL})$ at $65{ }^{\circ} \mathrm{C}$ and triethylamine $(2.5 \mathrm{~mL})$ was added. Borontrifluoride diethyletherate $(3.0 \mathrm{~mL})$ was then added after $1 \mathrm{~h}$, and the reaction mixture was further stirred at $65^{\circ} \mathrm{C}$ for $2 \mathrm{~h}$. The reaction was then poured into water, extracted with dichloromethane $(3 \times 25 \mathrm{~mL})$, dried over anhydrous $\mathrm{Na}_{2} \mathrm{SO}_{4}$, evaporated under vacuum. The crude product was purified by column chromatograph (silica gel, petroleum ether : dichloromethane $=6$ : 1) to give $1 \mathrm{a}(59 \mathrm{mg}, 77 \%) .{ }^{1} \mathrm{H} \mathrm{NMR}\left(500 \mathrm{MHz}, \mathrm{CDCl}_{3}, \mathrm{ppm}\right) \delta 8.61(\mathrm{~d}, J=7.0 \mathrm{~Hz}$, 2H), $8.07(\mathrm{~d}, J=8.5 \mathrm{~Hz}, 4 \mathrm{H}), 7.69$ (d, $J=8.5, \mathrm{~Hz}, 2 \mathrm{H}), 7.55$ (d, $J=8.5 \mathrm{~Hz}, 4 \mathrm{H}), 7.49$ 7.44 (m, 4H), 1.41 (s, 18H). ${ }^{13} \mathrm{C}$ NMR $\left(125 \mathrm{MHz}, \mathrm{CDCl}_{3}, \mathrm{ppm}\right) \delta 155.8,153.0,149.0$, 
148.7, 133.7, 130.9, 129.9, 129.1, 127.3, 126.1, 125.9, 125.7, 124.4, 34.9, 31.2. HRMS (APCI) calcd. for $\mathrm{C}_{40} \mathrm{H}_{35} \mathrm{BF}_{2} \mathrm{~N}_{3} \mathrm{~S}_{2}[\mathrm{M}+\mathrm{H}]^{+}: 670.2328$, found 670.2332 .

1b was prepared in $80 \%$ yield (79 $\mathrm{mg}$ ) from $\mathbf{2 b}(120 \mathrm{mg}, 0.106 \mathrm{mmol})$ using the above procedure. ${ }^{1} \mathrm{H}$ NMR (500 MHz, $\left.\mathrm{CDCl}_{3}, \mathrm{ppm}\right) \delta 8.57$ (s, 2H), $8.01(\mathrm{~d}, J=8.5 \mathrm{~Hz}, 4 \mathrm{H})$, $7.63(\mathrm{~s}, 2 \mathrm{H}), 7.43$ (d, $J=8.5 \mathrm{~Hz}, 4 \mathrm{H}), 6.94(\mathrm{~d}, J=8.5 \mathrm{~Hz}, 4 \mathrm{H}), 3.95$ (t, $J=6.5 \mathrm{~Hz}, 4 \mathrm{H})$, $1.83-1.77(\mathrm{~m}, 4 \mathrm{H}), 1.50-1.29(\mathrm{~m}, 36 \mathrm{H}), 0.89(\mathrm{t}, J=6.5 \mathrm{~Hz}, 6 \mathrm{H}) .{ }^{13} \mathrm{C}$ NMR (125 $\left.\mathrm{MHz}_{2} \mathrm{CDCl}_{3}, \mathrm{ppm}\right) \delta 160.4,148.7,148.4,131.6,130.5,128.4,127.2,126.0,125.8$, 124.6, 124.1, 115.0, 68.2, 60.4, 31.9, 29.6, 29.5, 29.4, 29.3, 26.1, 22.7, 14.1. HRMS (APCI) calcd. for $\mathrm{C}_{56} \mathrm{H}_{67} \mathrm{BF}_{2} \mathrm{~N}_{3} \mathrm{O}_{2} \mathrm{~S}_{2}[\mathrm{M}+\mathrm{H}]^{+}:$926.4730, found 926.4742 . 


\section{X-Ray Crystal packing}

(a)

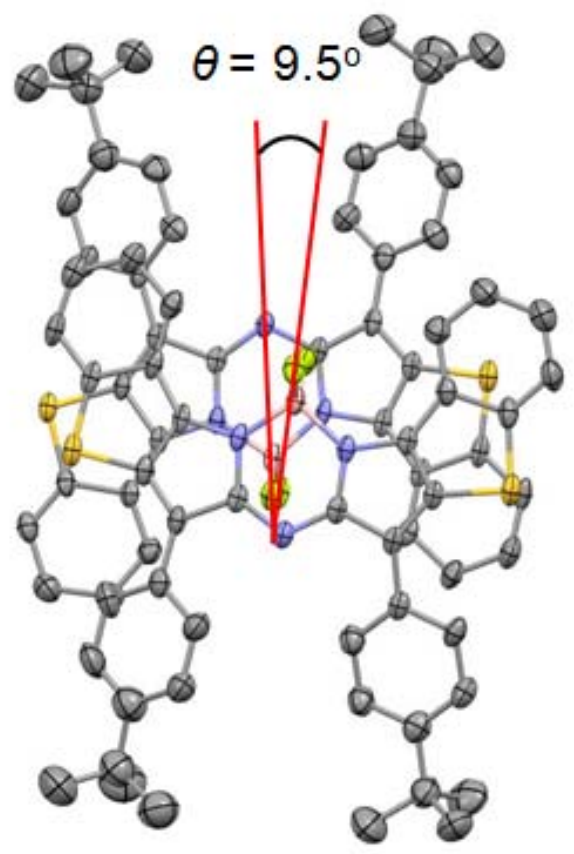

(b)

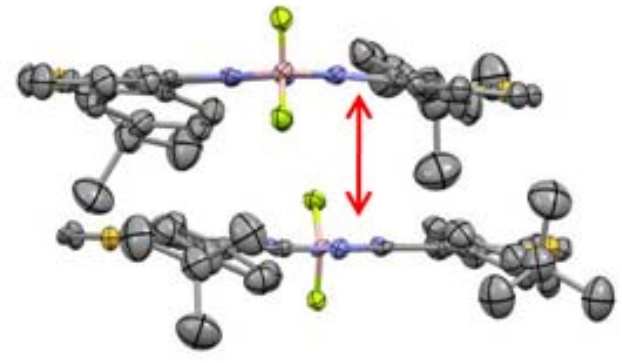

$$
d=3.82 \AA
$$

(c)

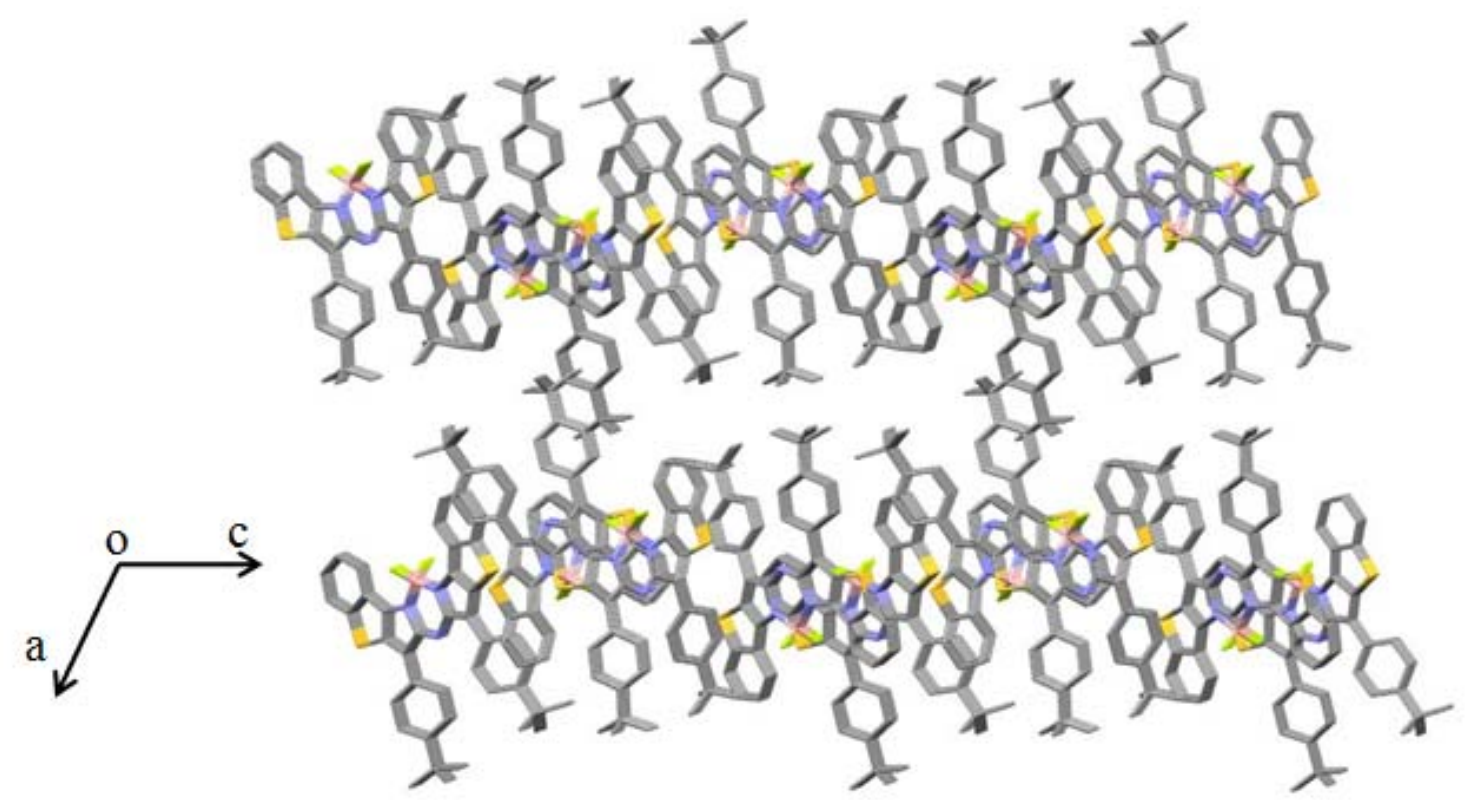

Figure S1. Side and top views of the dimer of $\mathbf{1 a}(a, b)$, the crystal packing viewed along the $b$ axis (c). 


\section{Photophysical properties}

Table S1: Photophysical properties of Aza-BODIPYs 4c, 1a and 1b in different solvents at room temperature.

\begin{tabular}{|c|c|c|c|c|c|c|}
\hline dyes & solvent & $\lambda_{\mathrm{abs}}^{\max }(\mathbf{n m})$ & $\varepsilon_{\text {abs }}^{\max }$ & $\lambda_{\mathrm{em}}^{\max }(\mathbf{n m})$ & $\Phi_{\mathrm{f}}$ & $\begin{array}{c}\text { Stokes-shift } \\
\text { (nm) }\end{array}$ \\
\hline & Toluene & 657 & 56300 & 683 & 0.25 & 26 \\
\hline \multirow[t]{3}{*}{$4 c$} & $\mathrm{CHCl}_{3}$ & 654 & 55500 & 680 & 0.25 & 26 \\
\hline & $\mathrm{THF}$ & 654 & 57000 & 681 & 0.15 & 27 \\
\hline & Toluene & 715 & 73300 & 796 & $<0.01$ & 81 \\
\hline \multirow[t]{3}{*}{$1 \mathrm{a}$} & $\mathrm{CHCl}_{3}$ & 711 & 77100 & 794 & $<0.01$ & 83 \\
\hline & $\mathrm{THF}$ & 709 & 67100 & 800 & $<0.01$ & 91 \\
\hline & Toluene & 733 & 84700 & 804 & $<0.01$ & 71 \\
\hline \multirow[t]{2}{*}{$1 \mathrm{~b}$} & $\mathrm{CHCl}_{3}$ & 731 & 86300 & 808 & $<0.01$ & 77 \\
\hline & THF & 729 & 79900 & 813 & $<0.01$ & 84 \\
\hline \multicolumn{7}{|c|}{$\begin{array}{l}\text { 4c was excited at } 610 \mathrm{~nm}, \mathbf{1 a} \text { was excited at } 680 \mathrm{~nm} \text {, 1b was excited at } 700 \mathrm{~nm} \text {; } \\
\text { Fluorescence quantum yields were calculated using } \\
\text { 3,5-di(4-methoxy)phenyl-1,7-diphenyl azaBODIPY }\left(\Phi_{\mathrm{f}}=0.36 \text { in chloroform) as the }\right. \\
\text { reference. }\end{array}$} \\
\hline
\end{tabular}



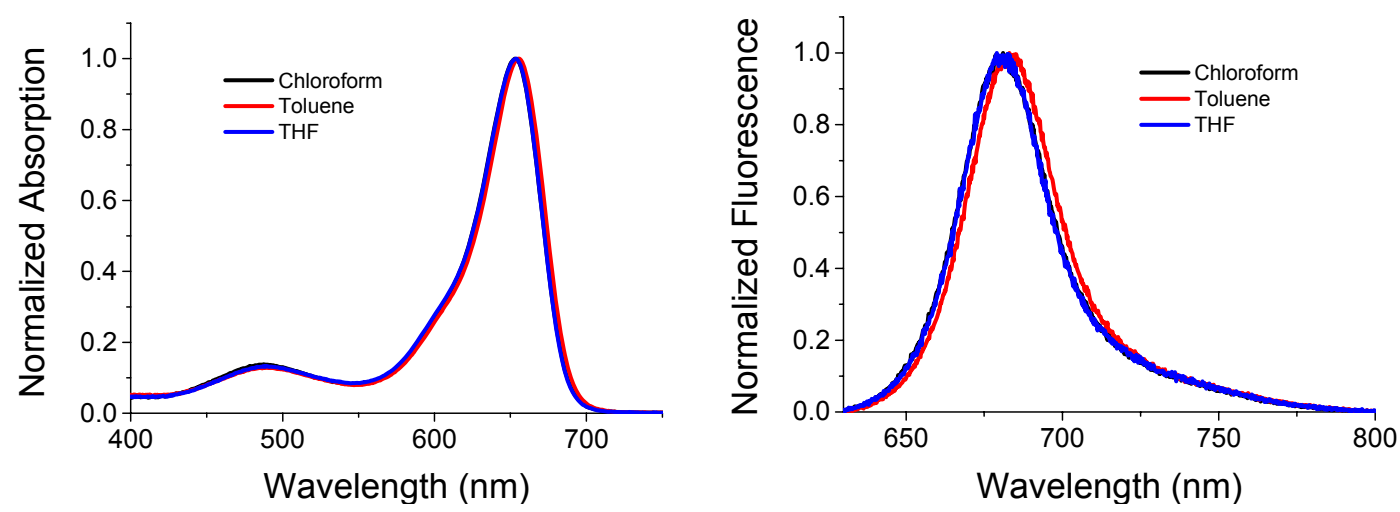

Figure S2. Normalized absorption (left) and emission (right) spectra of $\mathbf{4 c}$ in different solvents. Excited at $610 \mathrm{~nm}$.
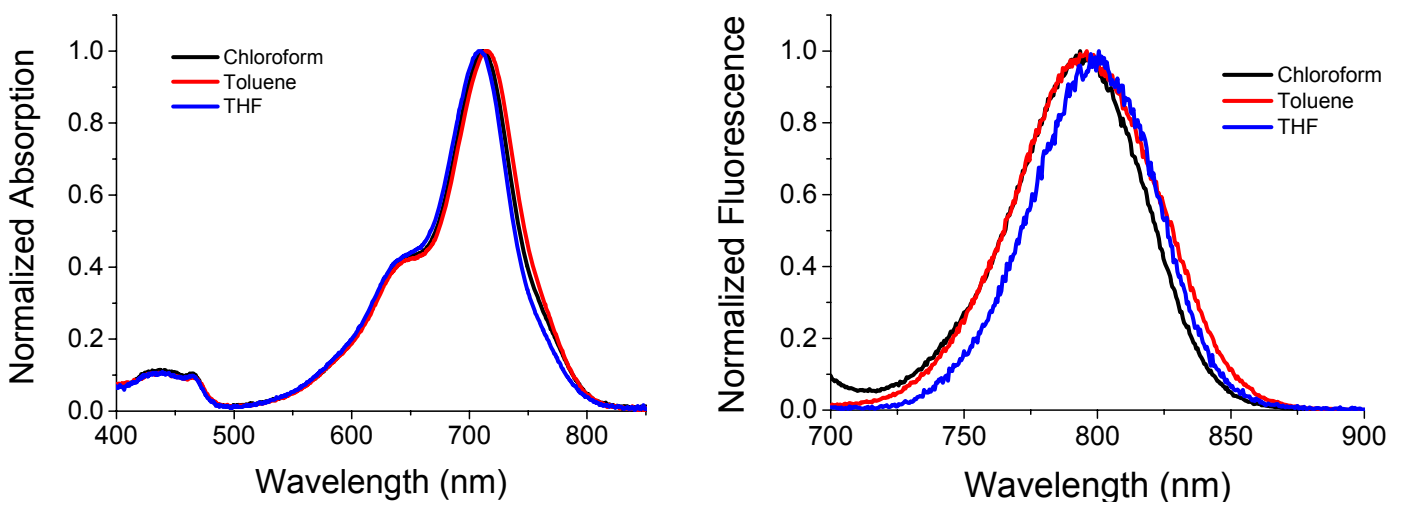

Figure S3. Normalized absorption (left) and emission (right) spectra of $\mathbf{1 a}$ in different solvents. Excited at $680 \mathrm{~nm}$.
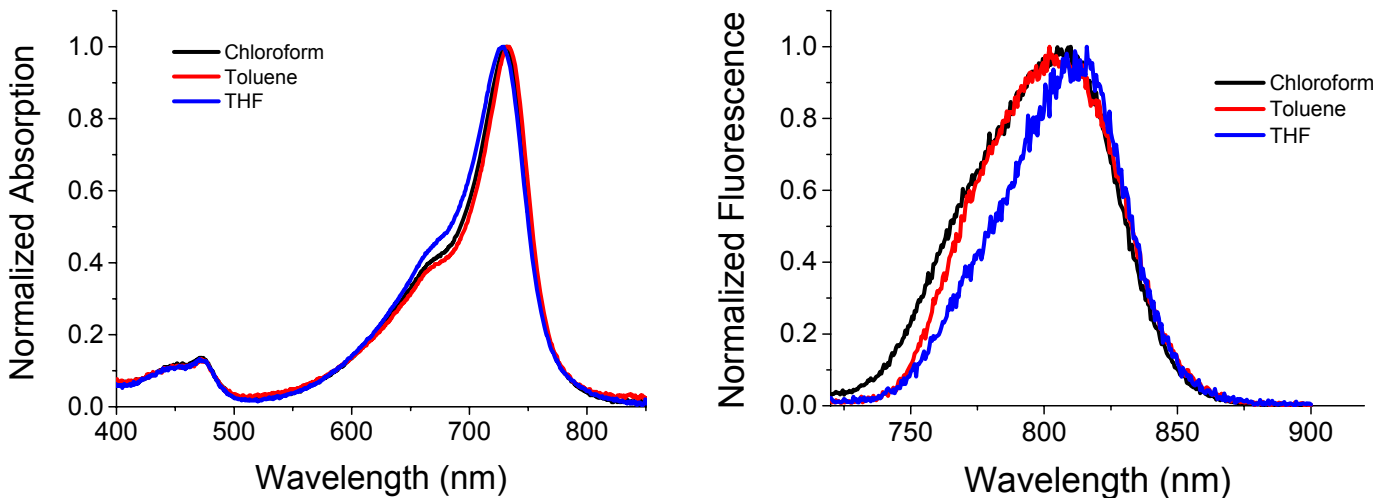

Figure S4. Normalized absorption (left) and emission (right) spectra of $\mathbf{1 b}$ in different solvents. Excited at $700 \mathrm{~nm}$. 


\section{Singlet oxygen properties}

The triplet states were determined by the chemical trapping method, since one molecule of singlet oxygen can react with one molecule of diphenylisobenzofuran (DPBF) which causes DPBF bleaching. Singlet oxygen quantum yields were measured at low dye concentrations (the irradiation wavelengths $>590 \mathrm{~nm} ; 2 \mathrm{~mW} / \mathrm{cm}^{2}$ ) to minimize the possibility of singlet oxygen quenching by the dyes. The photooxidation of DPBF was monitored between $10 \mathrm{~s}$ to $300 \mathrm{~s}$.
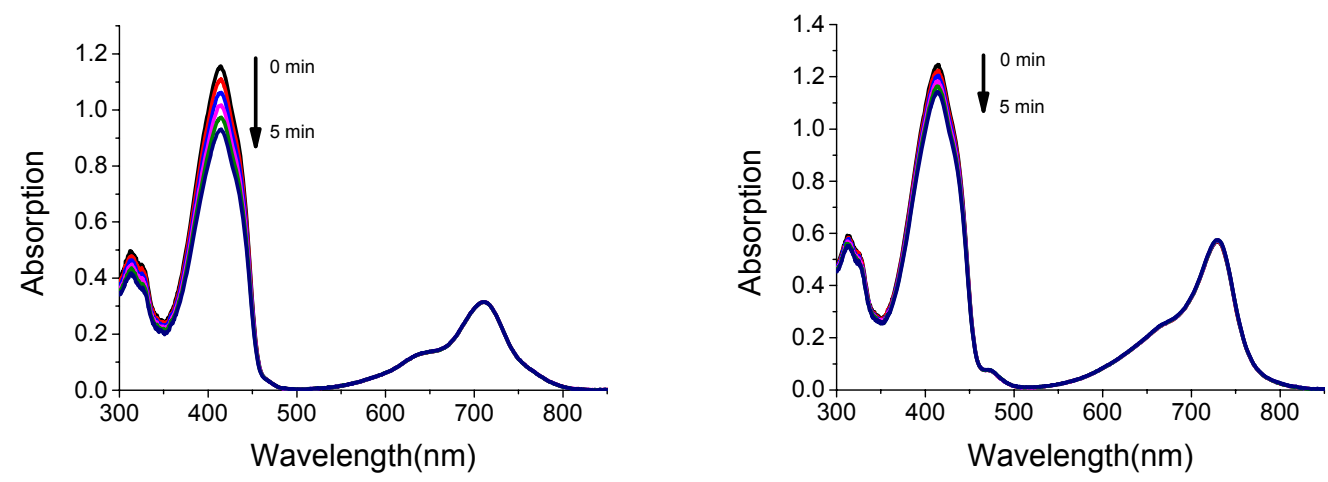

Figure S5. Absorption spectra of DPBF upon irradiation in the presence of 1a (left) and $\mathbf{1 b}$ (right) in $\mathrm{CH}_{2} \mathrm{Cl}_{2}\left(7.05 \times 10^{-6} \mathrm{M}\right)$ for $5 \mathrm{~min}$ (recorded at $1 \mathrm{~min}$ interval; $\lambda_{\text {irr }}>$ $\left.590 \mathrm{~nm} ; 2 \mathrm{~mW} / \mathrm{cm}^{2}\right)$. 


\section{Cyclic voltammograms of Aza-BODIPYs 4c and 1a.}

Cyclic voltammograms were measured in dichloromethane solution, containing $0.1 \mathrm{M}$ TBAPF $_{6}$ as the supporting electrolyte, glassy carbon electrode as a working electrode, Pt wire as a counter electrode, and saturated calomel electrode (SCE) as reference electrode at $100 \mathrm{mV} \mathrm{s}^{-1}$ of scaning rate at room temperature.

The HOMO energy level of $\mathbf{4 c}$ and $\mathbf{1 a}$ is estimated from HOMO $=-4.40-$ Eox $(\mathrm{eV})$ to be $-5.62 \mathrm{eV}$ and $-5.66 \mathrm{eV}$, respectively. The LUMO energy level of $\mathbf{4 c}$ and $\mathbf{1 a}$ is estimated from $\mathrm{LUMO}=-4.40-\mathrm{Ere}_{\mathrm{reV}}(\mathrm{eV})$ to be $-3.96 \mathrm{eV}$ and $-4.15 \mathrm{eV}$, respectively. The electrochemical bandgap of $\mathbf{4 c}$ and $\mathbf{1 a}$ are $1.66 \mathrm{eV}$ and $1.51 \mathrm{eV}$, respectively.

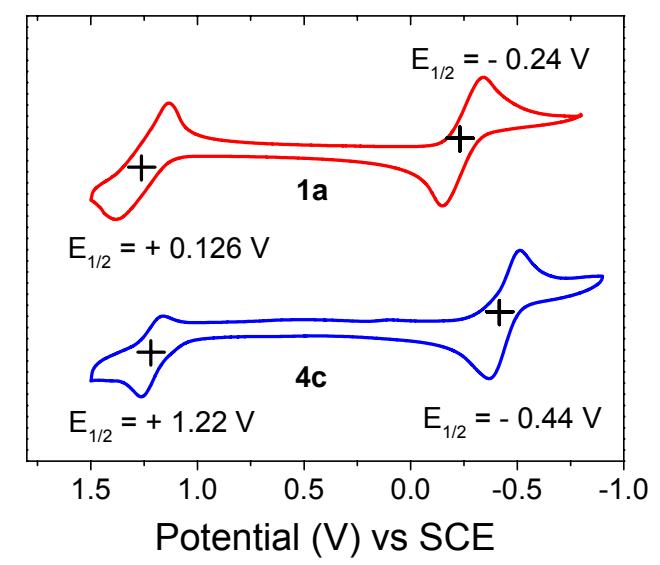

Figure S6. Cyclic voltammograms of $\mathbf{4 c}$ and $\mathbf{1 a}$ in $\mathrm{CH}_{2} \mathrm{Cl}_{2}$ with $0.1 \mathrm{M} \mathrm{nBu} 4 \mathrm{PF}_{6}$ as supporting electrolyte (scan rate: $100 \mathrm{mV} \mathrm{s}^{-1}$ ). 


\section{OFET Device Fabrication and Characterizations for $1 \mathrm{~b}$.}

Organic field-effect transistor (OFET) with top-gate/bottom-contact configuration was fabricated. Using the solution processing technique, the active layer was deposited by spin-casting solutions of $\mathbf{1 b}$ in trichloroethylene $\left(10 \mathrm{mg} \cdot \mathrm{mL}^{-1}\right)$ on patterned $\mathrm{Au}$ (source-drain) $/ \mathrm{SiO}_{2} / \mathrm{Si}$ substrates. After thermal annealing the semiconducting materials, a poly(perflurobutenylvinylether) (CYTOP) solution was spin-coated on top of it as the dielectric layer, followed by thermally evaporating a layer of aluminum as the gate electrode. ${ }^{8}$ All devices were fabricated in the glove box but tested under ambient conditions.
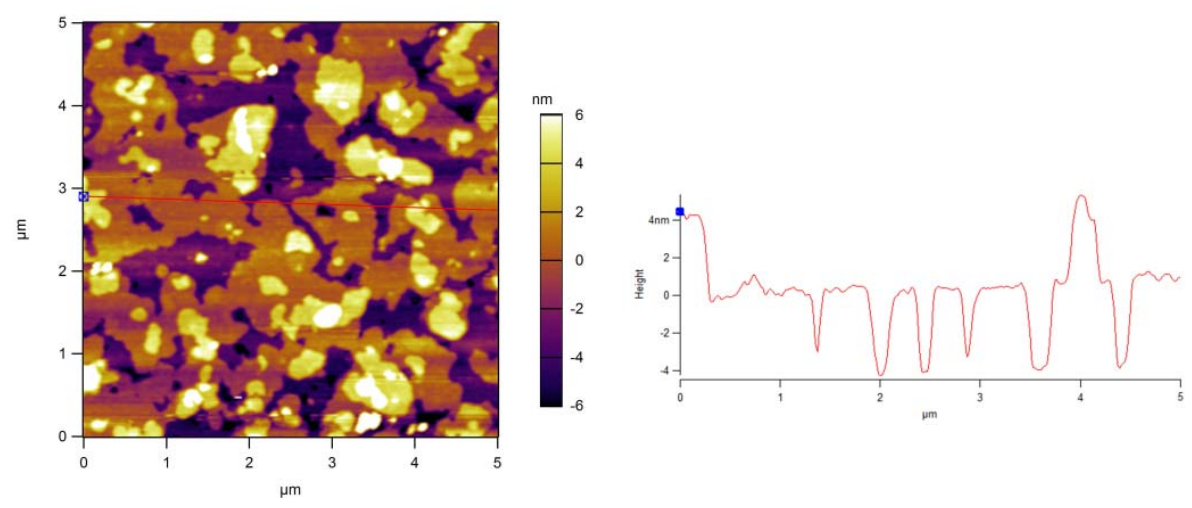

Figure S7. AFM height image of thin film of $\mathbf{1 b}$ upon thermal annealing at $140^{\circ} \mathrm{C}$.
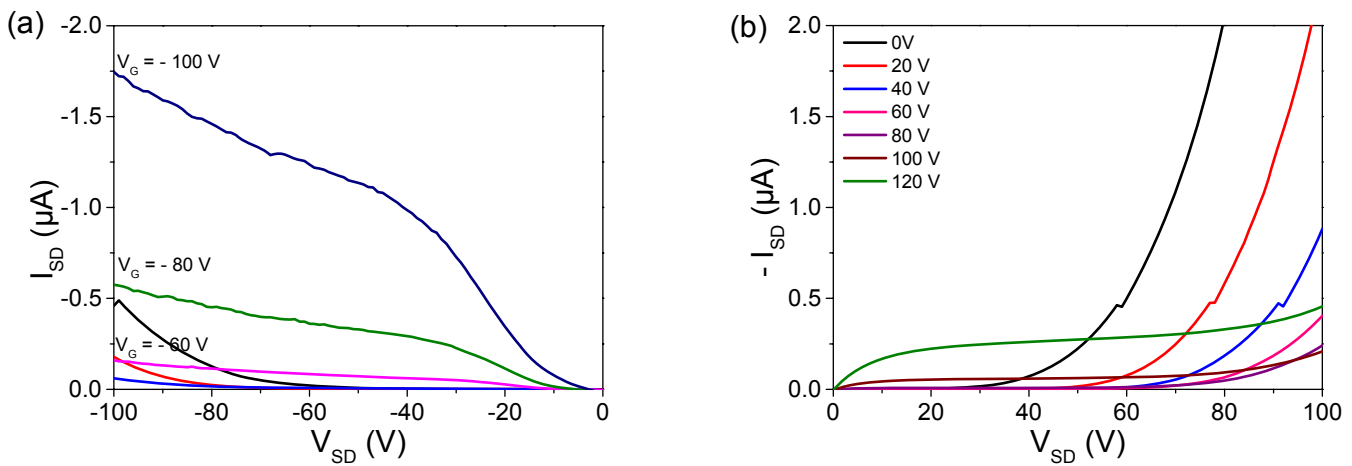

Figure S8. The output characteristic of $\mathbf{1 b}$ device 


\section{DFT calculations}

The ground state geometry was optimized by using DFT method at B3LYP/6-31G (d) level. The same method was used for vibrational analysis to verify that the optimized structures correspond to local minima on the energy surface. TD-DFT computations were used to obtain the vertical excitation energies and oscillator strengths at the optimized ground state equilibrium geometries under the B3LYP/6-31G (d) theoretical level. All the molecules in dichloromethane were done using the Self-Consistent Reaction Field (SCRF) method and Polarizable Continuum Model (PCM). All of the calculations were carried out by the methods implemented in Gaussian 09 package. ${ }^{9}$

Table S2. Selected electronic excitation energies $(\mathrm{eV})$ and oscillator strengths $(f)$, configurations of the low-lying excited states of the BODIPY $\mathbf{4 c}$ and 1 a calculated by TDDFT // B3LYP / 6-31G (d) based on the optimized ground state geometries. The TDDFT of all the molecules in dichloromethane were using the Self-Consistent Reaction Field (SCRF) method and the Polarizable Continuum Model (PCM).

\begin{tabular}{|c|c|c|c|c|c|c|}
\hline & \multirow{2}{*}{$\begin{array}{l}\text { Electronic } \\
\text { transition }\end{array}$} & \multicolumn{5}{|c|}{ TD//B3LYP/6-31G(d) } \\
\hline & & \multicolumn{2}{|c|}{ Energy/ eV [a] } & \multirow{2}{*}{$\frac{\boldsymbol{f}^{[\mathbf{b}]}}{0.8086}$} & \multirow{2}{*}{$\frac{\text { Composition }^{[\mathbf{c}]}}{\mathrm{HOMO} \rightarrow \text { LUMO }}$} & \multirow{2}{*}{$\frac{\text { CI I }^{[\mathrm{d}]}}{0.7005}$} \\
\hline \multirow{6}{*}{$4 c$} & $\mathrm{~S} 0 \rightarrow \mathrm{S} 1$ & $2.0439 \mathrm{eV}$ & $606.60 \mathrm{~nm}$ & & & \\
\hline & & & & & $\mathrm{HOMO} \leftarrow \mathrm{LUMO}$ & 0.1092 \\
\hline & $\mathrm{S} 0 \rightarrow \mathrm{S} 2$ & $2.4261 \mathrm{eV}$ & $511.05 \mathrm{~nm}$ & 0.0495 & $\mathrm{HOMO} \rightarrow$ LUMO & 0.1217 \\
\hline & & & & & HOMO $-1 \rightarrow$ LUMO & 0.6851 \\
\hline & $\mathrm{S} 0 \rightarrow \mathrm{S} 3$ & $2.5203 \mathrm{eV}$ & $491.95 \mathrm{~nm}$ & 0.4087 & HOMO - $1 \rightarrow$ LUMO & 0.1049 \\
\hline & & & & & HOMO $-2 \rightarrow$ LUMO & 0.6953 \\
\hline \multirow{6}{*}{$1 \mathbf{a}$} & $\mathrm{S} 0 \rightarrow \mathrm{S} 1$ & $1.8321 \mathrm{eV}$ & $676.73 \mathrm{~nm}$ & 0.1184 & $\mathrm{HOMO} \rightarrow \mathrm{LUMO}$ & 0.2462 \\
\hline & & & & & HOMO $-1 \rightarrow$ LUMO & 0.6622 \\
\hline & $\mathrm{S} 0 \rightarrow \mathrm{S} 2$ & $1.9716 \mathrm{eV}$ & $628.85 \mathrm{~nm}$ & 0.9021 & $\mathrm{HOMO} \rightarrow \mathrm{LUMO}$ & 0.6669 \\
\hline & & & & & $\mathrm{HOMO} \leftarrow \mathrm{LUMO}$ & 0.1156 \\
\hline & & & & & HOMO $-1 \rightarrow$ LUMO & 0.2406 \\
\hline & $\mathrm{S} 0 \rightarrow \mathrm{S} 3$ & $1.9956 \mathrm{eV}$ & $621.28 \mathrm{~nm}$ & 0.1952 & HOMO $-2 \rightarrow$ LUMO & 0.7015 \\
\hline
\end{tabular}

[a] Only the selected low-lying excited states are presented. [b] Oscillator strength. [c] Only the main configurations are presented. [d] The CI coefficients are in absolute values. 


\section{DFT optimized coordinates}

\section{Compound 4c}

\begin{tabular}{|c|c|c|c|}
\hline F & 4.08109300 & -0.01465800 & 0.70393300 \\
\hline $\mathrm{F}$ & 3.66725800 & -0.00266500 & -1.56109700 \\
\hline $\mathrm{N}$ & 2.18132700 & 1.25302200 & -0.12734700 \\
\hline $\mathrm{N}$ & 0.12566300 & 0.01327000 & -0.16230400 \\
\hline $\mathrm{N}$ & 2.16499200 & -1.25215000 & -0.13471200 \\
\hline $\mathrm{C}$ & 2.49948300 & 2.56800900 & 0.03163200 \\
\hline $\mathrm{C}$ & -5.47385400 & -3.95307200 & 0.14013500 \\
\hline $\mathrm{C}$ & 1.30506700 & 3.32960300 & 0.09889700 \\
\hline $\mathrm{C}$ & 0.21947300 & 2.47452100 & -0.04163500 \\
\hline $\mathrm{C}$ & -6.27147000 & -3.01990200 & -0.80217100 \\
\hline $\mathrm{C}$ & -5.72784400 & -5.40816200 & -0.29771600 \\
\hline $\mathrm{C}$ & 0.78966700 & 1.15701600 & -0.15873100 \\
\hline $\mathrm{C}$ & -5.99604900 & -3.78659100 & 1.58744400 \\
\hline $\mathrm{C}$ & 0.77316100 & -1.13859500 & -0.12439600 \\
\hline $\mathrm{C}$ & 0.18934100 & -2.45165500 & -0.00824900 \\
\hline $\mathrm{C}$ & 1.26937000 & -3.32167600 & 0.06830600 \\
\hline $\mathrm{C}$ & 2.47078500 & -2.57352600 & -0.01151900 \\
\hline $\mathrm{C}$ & 3.84890300 & 3.13840300 & 0.10441600 \\
\hline $\mathrm{C}$ & 4.04936600 & 4.27524600 & 0.91292100 \\
\hline $\mathrm{C}$ & 5.29807600 & 4.88395900 & 0.99147600 \\
\hline $\mathrm{C}$ & 6.37046900 & 4.37652300 & 0.25440300 \\
\hline $\mathrm{C}$ & 6.18146800 & 3.25588300 & -0.55675100 \\
\hline $\mathrm{C}$ & 4.93654100 & 2.63680900 & -0.63445200 \\
\hline $\mathrm{C}$ & -1.19930600 & 2.83061100 & -0.05022000 \\
\hline $\mathrm{C}$ & -2.16587500 & 2.05138500 & -0.71527800 \\
\hline $\mathrm{C}$ & -3.50304100 & 2.42989000 & -0.72020400 \\
\hline $\mathrm{C}$ & -3.95247800 & 3.59349900 & -0.07060300 \\
\hline $\mathrm{C}$ & -2.98425900 & 4.36563700 & 0.58783700 \\
\hline $\mathrm{C}$ & -1.64144800 & 3.99601800 & 0.59786400 \\
\hline $\mathrm{C}$ & -5.44413300 & 3.96778600 & -0.10275900 \\
\hline $\mathrm{C}$ & -5.89128500 & 4.17074200 & -1.57052600 \\
\hline $\mathrm{C}$ & -6.27458200 & 2.82646900 & 0.53196800 \\
\hline $\mathrm{C}$ & -5.74093400 & 5.26506700 & 0.67328800 \\
\hline $\mathrm{C}$ & 3.81460300 & -3.16076900 & 0.04301100 \\
\hline $\mathrm{C}$ & 4.00816100 & -4.30998200 & 0.83580800 \\
\hline $\mathrm{C}$ & 5.25030900 & -4.93394900 & 0.89708100 \\
\hline $\mathrm{C}$ & 6.32289000 & -4.43035500 & 0.15772200 \\
\hline $\mathrm{C}$ & 6.14065800 & -3.29757800 & -0.63776500 \\
\hline $\mathrm{C}$ & 4.90253100 & -2.66285300 & -0.69773000 \\
\hline $\mathrm{C}$ & -1.23174900 & -2.80457500 & 0.01667300 \\
\hline $\mathrm{C}$ & -1.64210600 & -4.09615400 & -0.35573500 \\
\hline $\mathrm{C}$ & -2.98284200 & -4.47093800 & -0.32030600 \\
\hline
\end{tabular}




\begin{tabular}{|c|c|c|c|}
\hline $\mathrm{C}$ & -3.98344300 & -3.57723900 & 0.08807900 \\
\hline $\mathrm{C}$ & -3.56725000 & -2.28560500 & 0.45665600 \\
\hline $\mathrm{C}$ & -2.23216300 & -1.90091300 & 0.42544300 \\
\hline B & 3.11014100 & -0.00474100 & -0.27055700 \\
\hline $\mathrm{H}$ & -6.16955100 & -1.96806600 & -0.51536400 \\
\hline $\mathrm{H}$ & -7.33870300 & -3.27188300 & -0.77422300 \\
\hline $\mathrm{H}$ & -5.92540300 & -3.11956200 & -1.83728700 \\
\hline $\mathrm{H}$ & -5.40712500 & -5.58553300 & -1.33054200 \\
\hline $\mathrm{H}$ & -6.80000000 & -5.62807000 & -0.24291400 \\
\hline $\mathrm{H}$ & -5.21045100 & -6.12543400 & 0.34957300 \\
\hline $\mathrm{H}$ & -5.45222900 & -4.44091400 & 2.27824000 \\
\hline $\mathrm{H}$ & -7.06063800 & -4.04579800 & 1.64015000 \\
\hline $\mathrm{H}$ & -5.88531200 & -2.75771700 & 1.94511600 \\
\hline $\mathrm{H}$ & 3.22483200 & 4.66140400 & 1.50470800 \\
\hline $\mathrm{H}$ & 5.43535200 & 5.75115200 & 1.63165100 \\
\hline $\mathrm{H}$ & 7.34618900 & 4.85143500 & 0.31244700 \\
\hline $\mathrm{H}$ & 7.00948000 & 2.86013500 & -1.13848600 \\
\hline $\mathrm{H}$ & -1.85957900 & 1.14996100 & -1.23274800 \\
\hline $\mathrm{H}$ & -4.21220900 & 1.80225900 & -1.25281800 \\
\hline $\mathrm{H}$ & -3.27116100 & 5.27020900 & 1.11226600 \\
\hline $\mathrm{H}$ & -0.92751700 & 4.60970300 & 1.14028700 \\
\hline $\mathrm{H}$ & -5.32443500 & 4.98003300 & -2.04454500 \\
\hline $\mathrm{H}$ & -6.95599100 & 4.43126600 & -1.61156500 \\
\hline $\mathrm{H}$ & -5.74598300 & 3.26590400 & -2.16953900 \\
\hline $\mathrm{H}$ & -6.13971000 & 1.87904300 & -0.00025200 \\
\hline $\mathrm{H}$ & -7.34293000 & 3.07364800 & 0.50694700 \\
\hline $\mathrm{H}$ & -5.98684300 & 2.66710000 & 1.57744200 \\
\hline $\mathrm{H}$ & -5.47105300 & 5.17867300 & 1.73202300 \\
\hline $\mathrm{H}$ & -6.81272900 & 5.48723200 & 0.62325400 \\
\hline $\mathrm{H}$ & -5.20639800 & 6.12434100 & 0.25248800 \\
\hline $\mathrm{H}$ & 3.18483200 & -4.69365100 & 1.43071900 \\
\hline $\mathrm{H}$ & 5.38225200 & -5.81009300 & 1.52610400 \\
\hline $\mathrm{H}$ & 7.29334700 & -4.91736100 & 0.20221300 \\
\hline $\mathrm{H}$ & 6.96868600 & -2.90397100 & -1.22095000 \\
\hline $\mathrm{H}$ & -0.90421700 & -4.81346500 & -0.70378700 \\
\hline $\mathrm{H}$ & -3.24152600 & -5.47872000 & -0.62575000 \\
\hline $\mathrm{H}$ & -4.30233900 & -1.55534500 & 0.78423700 \\
\hline $\mathrm{H}$ & -1.95432500 & -0.89666400 & 0.72106200 \\
\hline $\mathrm{H}$ & 1.22870700 & -4.39689700 & 0.16616000 \\
\hline $\mathrm{H}$ & 1.27377000 & 4.40790300 & 0.16269100 \\
\hline $\mathrm{H}$ & 4.79707400 & 1.77842700 & -1.27845800 \\
\hline $\mathrm{H}$ & 4.76882100 & -1.79399800 & -1.32861800 \\
\hline
\end{tabular}




\section{Compound 1a}

\begin{tabular}{|c|c|c|c|}
\hline S & -1.58935200 & -5.05176000 & -0.04507500 \\
\hline S & -1.61297400 & 5.04625600 & -0.08941100 \\
\hline $\mathrm{F}$ & -3.61598400 & -0.00052700 & 1.52295000 \\
\hline $\mathrm{F}$ & -4.10439500 & -0.01245400 & -0.70698400 \\
\hline $\mathrm{N}$ & -2.14493700 & -1.23550300 & 0.04455700 \\
\hline $\mathrm{N}$ & -0.09346800 & 0.00239300 & 0.06189700 \\
\hline $\mathrm{N}$ & -2.15183300 & 1.22842000 & 0.02971000 \\
\hline $\mathrm{C}$ & -2.45087600 & -2.55356300 & 0.02446600 \\
\hline $\mathrm{C}$ & 5.50217200 & 3.90922700 & 0.12942200 \\
\hline $\mathrm{C}$ & -1.26037500 & -3.32744500 & 0.01087900 \\
\hline $\mathrm{C}$ & -0.16102800 & -2.47176800 & 0.00177200 \\
\hline $\mathrm{C}$ & 6.31558900 & 2.81291500 & -0.59989700 \\
\hline $\mathrm{C}$ & 5.80293500 & 5.26008700 & -0.54701400 \\
\hline $\mathrm{C}$ & -0.74995800 & -1.14594000 & 0.03466500 \\
\hline $\mathrm{C}$ & 5.96532700 & 3.99399800 & 1.60385600 \\
\hline $\mathrm{C}$ & -0.75664600 & 1.14719000 & 0.06338300 \\
\hline $\mathrm{C}$ & -0.17434500 & 2.47537300 & 0.02337100 \\
\hline $\mathrm{C}$ & -1.27680000 & 3.32290800 & -0.05482300 \\
\hline $\mathrm{C}$ & -2.46391900 & 2.54321800 & -0.04309000 \\
\hline $\mathrm{C}$ & -3.65612800 & -3.33144000 & -0.00498300 \\
\hline $\mathrm{C}$ & -5.00544600 & -2.93226500 & -0.02298100 \\
\hline $\mathrm{C}$ & -5.99840700 & -3.90307100 & -0.04482800 \\
\hline $\mathrm{C}$ & -5.66928900 & -5.26786200 & -0.05693000 \\
\hline $\mathrm{C}$ & -4.33865900 & -5.68560500 & -0.05499200 \\
\hline $\mathrm{C}$ & -3.33760400 & -4.71726300 & -0.03216900 \\
\hline $\mathrm{C}$ & 1.26014100 & -2.81017300 & -0.02950800 \\
\hline $\mathrm{C}$ & 2.23182000 & -1.93187500 & -0.55027300 \\
\hline $\mathrm{C}$ & 3.57218900 & -2.29420200 & -0.58064100 \\
\hline $\mathrm{C}$ & 4.02352000 & -3.53715100 & -0.10083400 \\
\hline $\mathrm{C}$ & 3.05207900 & -4.40516000 & 0.41753400 \\
\hline $\mathrm{C}$ & 1.70444700 & -4.05414400 & 0.45172900 \\
\hline $\mathrm{C}$ & 5.51873500 & -3.89090000 & -0.16237900 \\
\hline $\mathrm{C}$ & 5.98749100 & -3.88085500 & -1.63735900 \\
\hline $\mathrm{C}$ & 6.32965600 & -2.84416900 & 0.63885300 \\
\hline $\mathrm{C}$ & 5.81660200 & -5.28260900 & 0.42719700 \\
\hline $\mathrm{C}$ & -3.67232600 & 3.31613400 & -0.08121200 \\
\hline $\mathrm{C}$ & -5.01973900 & 2.91117900 & -0.10977900 \\
\hline $\mathrm{C}$ & -6.01671900 & 3.87766800 & -0.13909500 \\
\hline $\mathrm{C}$ & -5.69348800 & 5.24386800 & -0.14727200 \\
\hline $\mathrm{C}$ & -4.36475600 & 5.66737300 & -0.13319200 \\
\hline $\mathrm{C}$ & -3.35967100 & 4.70347300 & -0.10111700 \\
\hline $\mathrm{C}$ & 1.24541700 & 2.81898500 & 0.04107000 \\
\hline
\end{tabular}




\begin{tabular}{|c|c|c|c|}
\hline $\mathrm{C}$ & 1.69290200 & 4.02511300 & -0.52546400 \\
\hline $\mathrm{C}$ & 3.04013800 & 4.37896900 & -0.50473200 \\
\hline $\mathrm{C}$ & 4.00711700 & 3.55177500 & 0.08407800 \\
\hline $\mathrm{C}$ & 3.55218900 & 2.34701900 & 0.65041400 \\
\hline $\mathrm{C}$ & 2.21218500 & 1.98241100 & 0.63456000 \\
\hline B & -3.08587300 & -0.00461900 & 0.24524500 \\
\hline $\mathrm{H}$ & 6.17896200 & 1.82871700 & -0.13947900 \\
\hline $\mathrm{H}$ & 7.38611200 & 3.04961300 & -0.56838500 \\
\hline $\mathrm{H}$ & 6.01529500 & 2.73568900 & -1.65107600 \\
\hline $\mathrm{H}$ & 5.52286700 & 5.25747900 & -1.60661800 \\
\hline $\mathrm{H}$ & 6.87683200 & 5.46988800 & -0.49061300 \\
\hline $\mathrm{H}$ & 5.27906700 & 6.08799600 & -0.05592300 \\
\hline $\mathrm{H}$ & 5.41107400 & 4.76919400 & 2.14495600 \\
\hline $\mathrm{H}$ & 7.03275000 & 4.24146500 & 1.65349600 \\
\hline $\mathrm{H}$ & 5.81837200 & 3.04708100 & 2.13370200 \\
\hline $\mathrm{H}$ & -5.25158500 & -1.87741200 & -0.03741200 \\
\hline $\mathrm{H}$ & -7.04178300 & -3.60215600 & -0.05733100 \\
\hline $\mathrm{H}$ & -6.46005000 & -6.01275200 & -0.07402300 \\
\hline $\mathrm{H}$ & -4.08996700 & -6.74262300 & -0.07325400 \\
\hline $\mathrm{H}$ & 1.92701000 & -0.96606800 & -0.93399700 \\
\hline $\mathrm{H}$ & 4.28353000 & -1.58734800 & -0.99859900 \\
\hline $\mathrm{H}$ & 3.33839500 & -5.37444200 & 0.81011700 \\
\hline $\mathrm{H}$ & 0.99341900 & -4.74788900 & 0.89126400 \\
\hline $\mathrm{H}$ & 5.43521900 & -4.61954000 & -2.22924300 \\
\hline $\mathrm{H}$ & 7.05506300 & -4.12510200 & -1.69878600 \\
\hline $\mathrm{H}$ & 5.84228700 & -2.90162300 & -2.10562200 \\
\hline $\mathrm{H}$ & 6.20011200 & -1.83346600 & 0.23778300 \\
\hline $\mathrm{H}$ & 7.39962700 & -3.08255000 & 0.60131300 \\
\hline $\mathrm{H}$ & 6.02109200 & -2.82959800 & 1.69034800 \\
\hline $\mathrm{H}$ & 5.52945900 & -5.34882300 & 1.48285900 \\
\hline $\mathrm{H}$ & 6.89113000 & -5.48774300 & 0.36429500 \\
\hline $\mathrm{H}$ & 5.29706500 & -6.07747400 & -0.11990100 \\
\hline $\mathrm{H}$ & -5.26033000 & 1.85511300 & -0.12818900 \\
\hline $\mathrm{H}$ & -7.05862100 & 3.57218900 & -0.16067400 \\
\hline $\mathrm{H}$ & -6.48729800 & 5.98535000 & -0.17044000 \\
\hline $\mathrm{H}$ & -4.12055800 & 6.72550400 & -0.14717000 \\
\hline $\mathrm{H}$ & 0.98485800 & 4.68400600 & -1.02011300 \\
\hline $\mathrm{H}$ & 3.32995600 & 5.31730900 & -0.96412600 \\
\hline $\mathrm{H}$ & 4.26009100 & 1.67448800 & 1.12671000 \\
\hline $\mathrm{H}$ & 1.90337500 & 1.04781600 & 1.08671500 \\
\hline
\end{tabular}




\section{9. ${ }^{1} \mathrm{H}$ NMR and ${ }^{13} \mathrm{C}$ NMR spectra for the compounds}

${ }^{1} \mathrm{H}$ NMR and ${ }^{13} \mathrm{C}$ NMR spectrum of $\mathbf{6 a}$ in $\mathrm{CDCl}_{3}$ solution

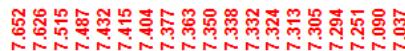
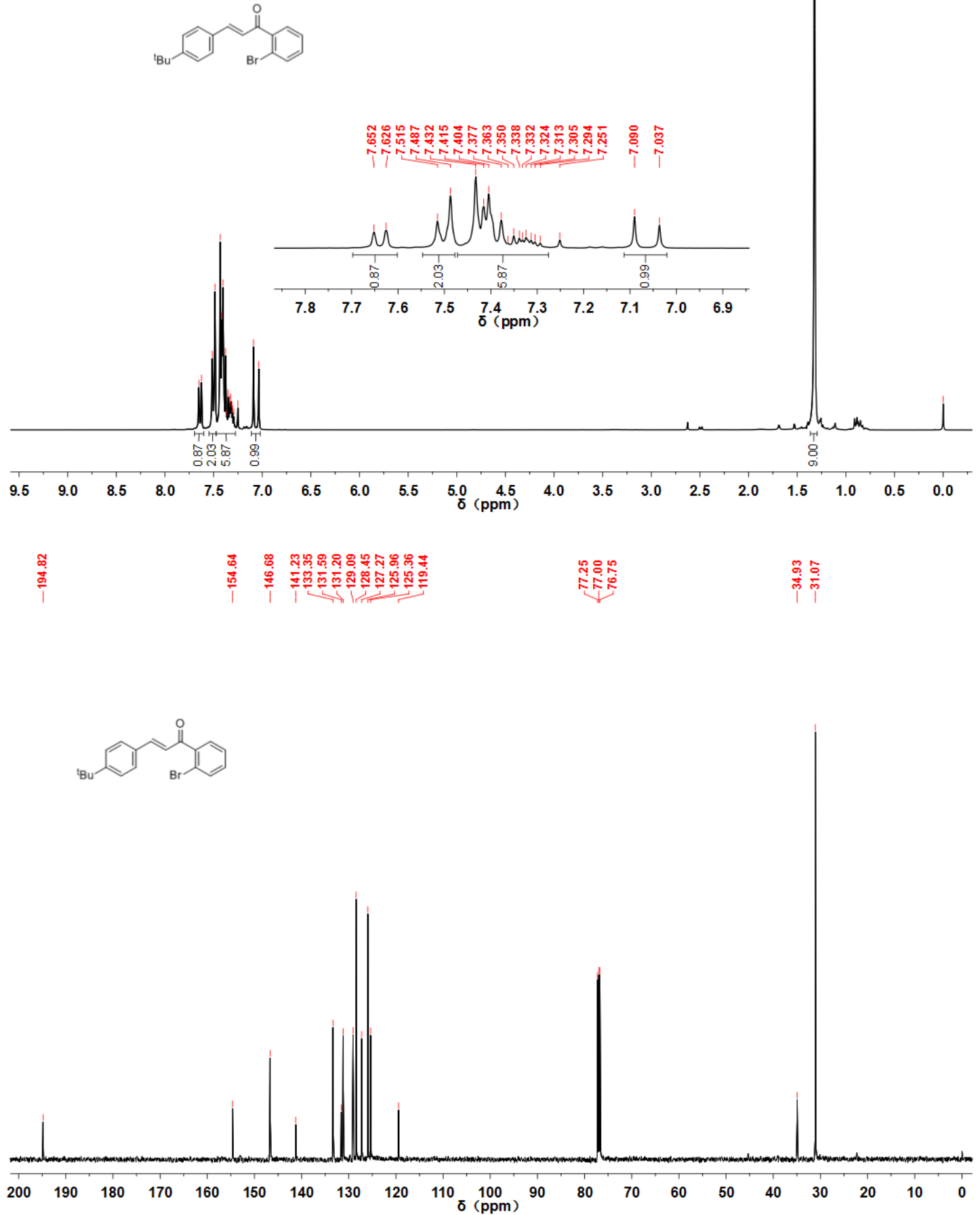
${ }^{1} \mathrm{H}$ NMR and ${ }^{13} \mathrm{C}$ NMR spectrum of $\mathbf{6 b}$ in $\mathrm{CDCl}_{3}$ solution

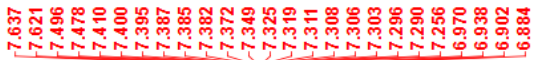

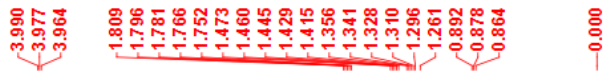
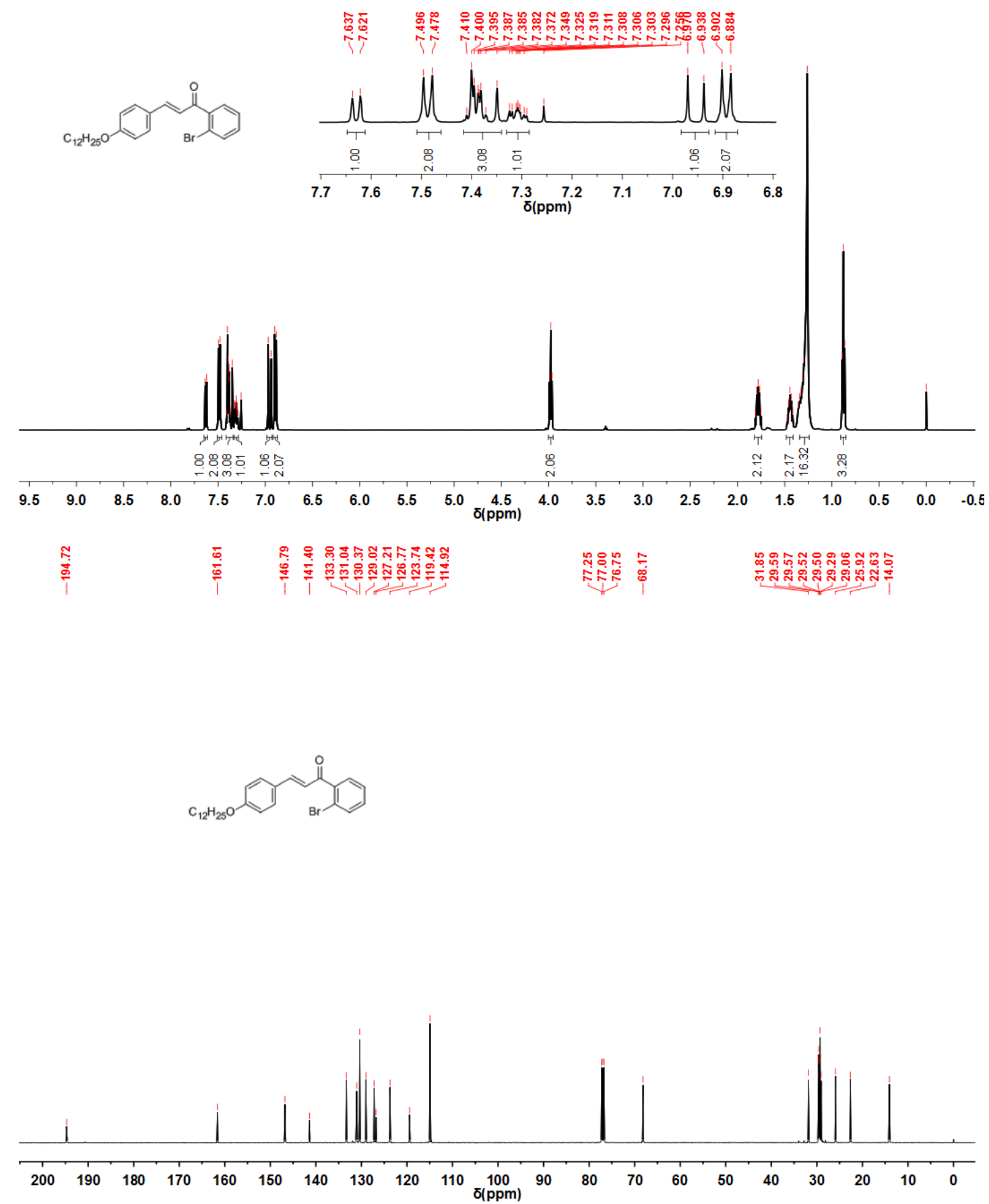
${ }^{1} \mathrm{H}$ NMR and ${ }^{13} \mathrm{C}$ NMR spectrum of $\mathbf{6 c}$ in $\mathrm{CDCl}_{3}$ solution

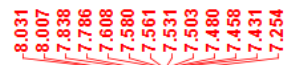
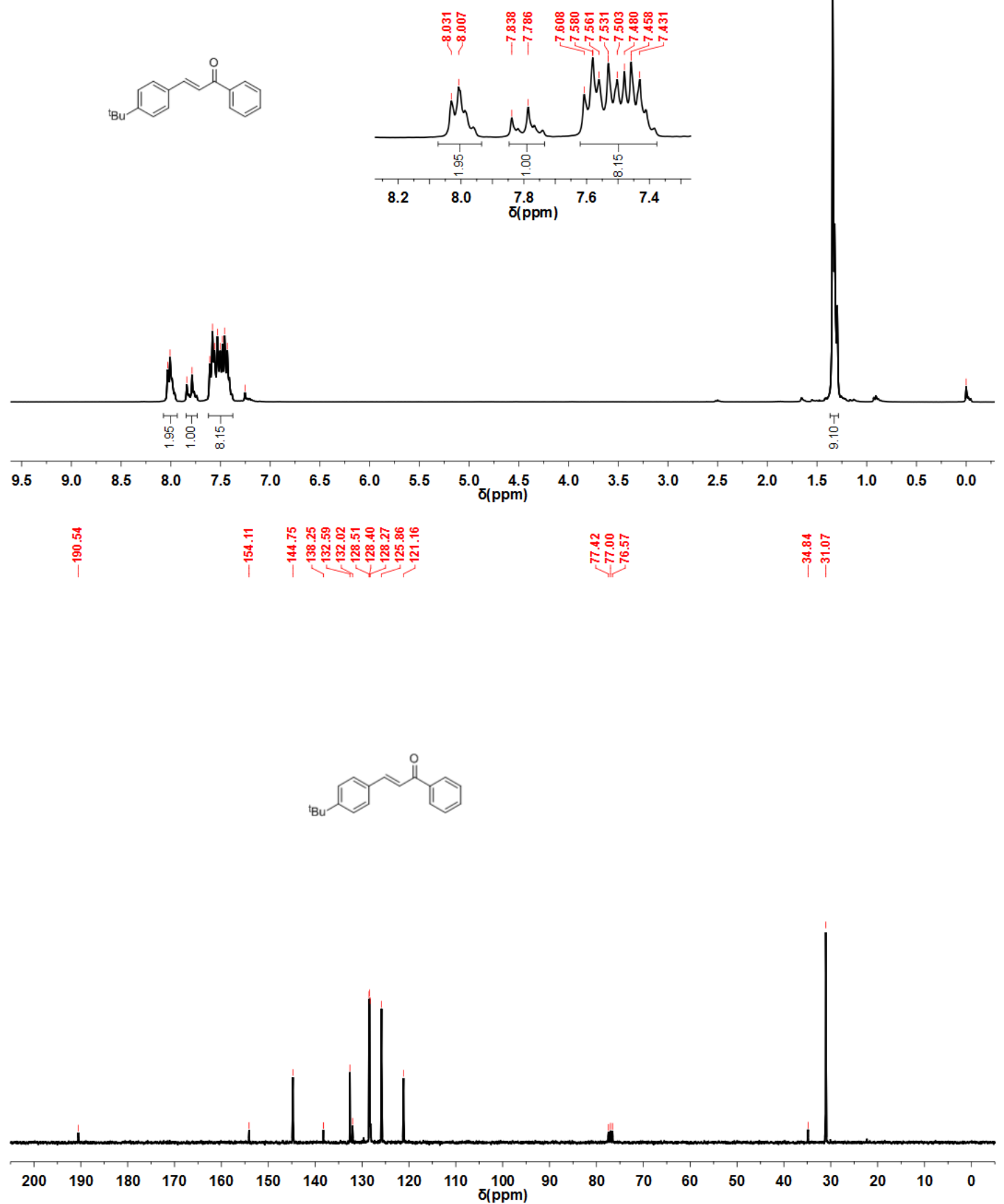
${ }^{1} \mathrm{H}$ NMR and ${ }^{13} \mathrm{C}$ NMR spectrum of $\mathbf{5 a}$ in $\mathrm{CDCl}_{3}$ solution
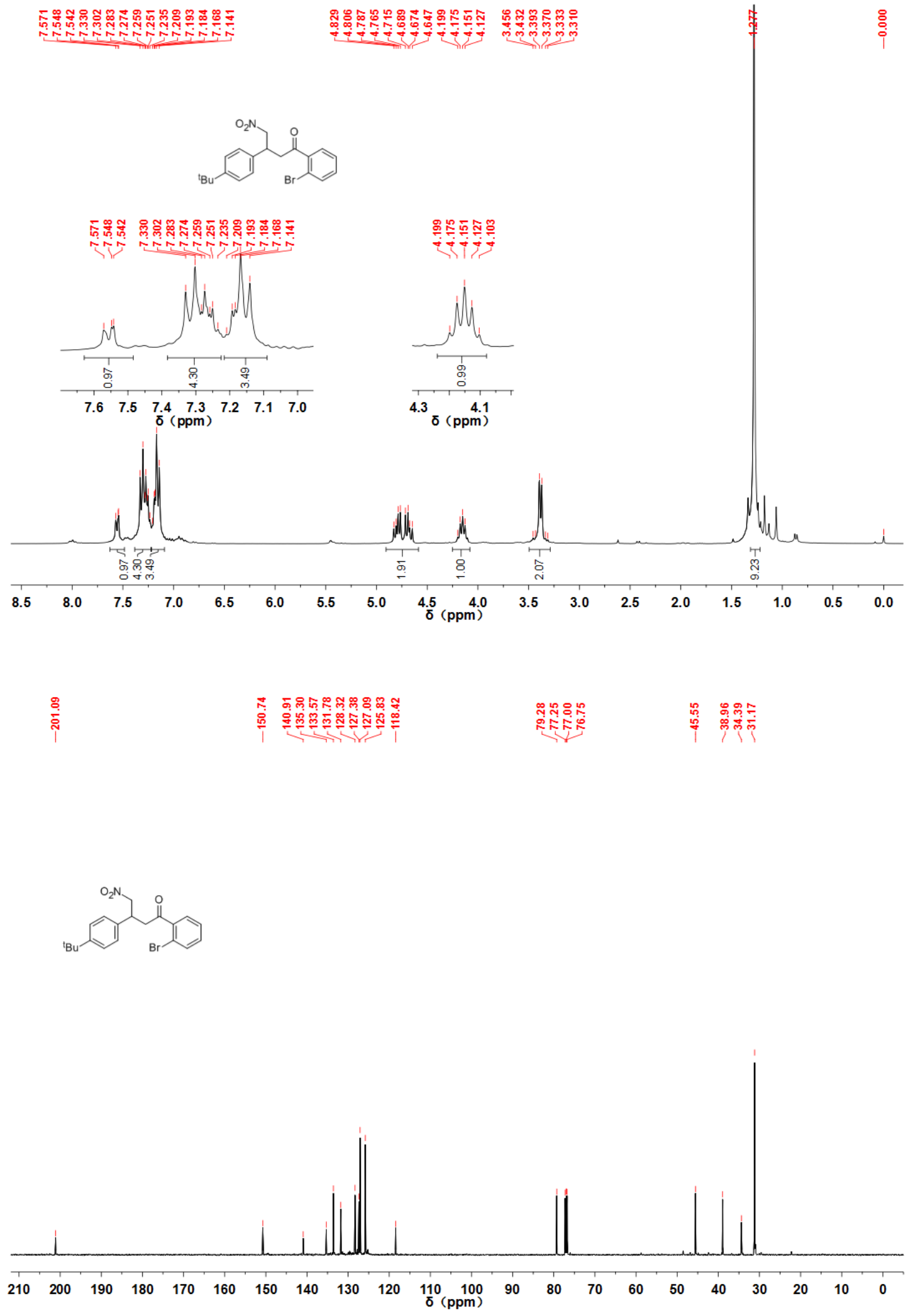
${ }^{1} \mathrm{H}$ NMR and ${ }^{13} \mathrm{C}$ NMR spectrum of $\mathbf{5 b}$ in $\mathrm{CDCl}_{3}$ solution
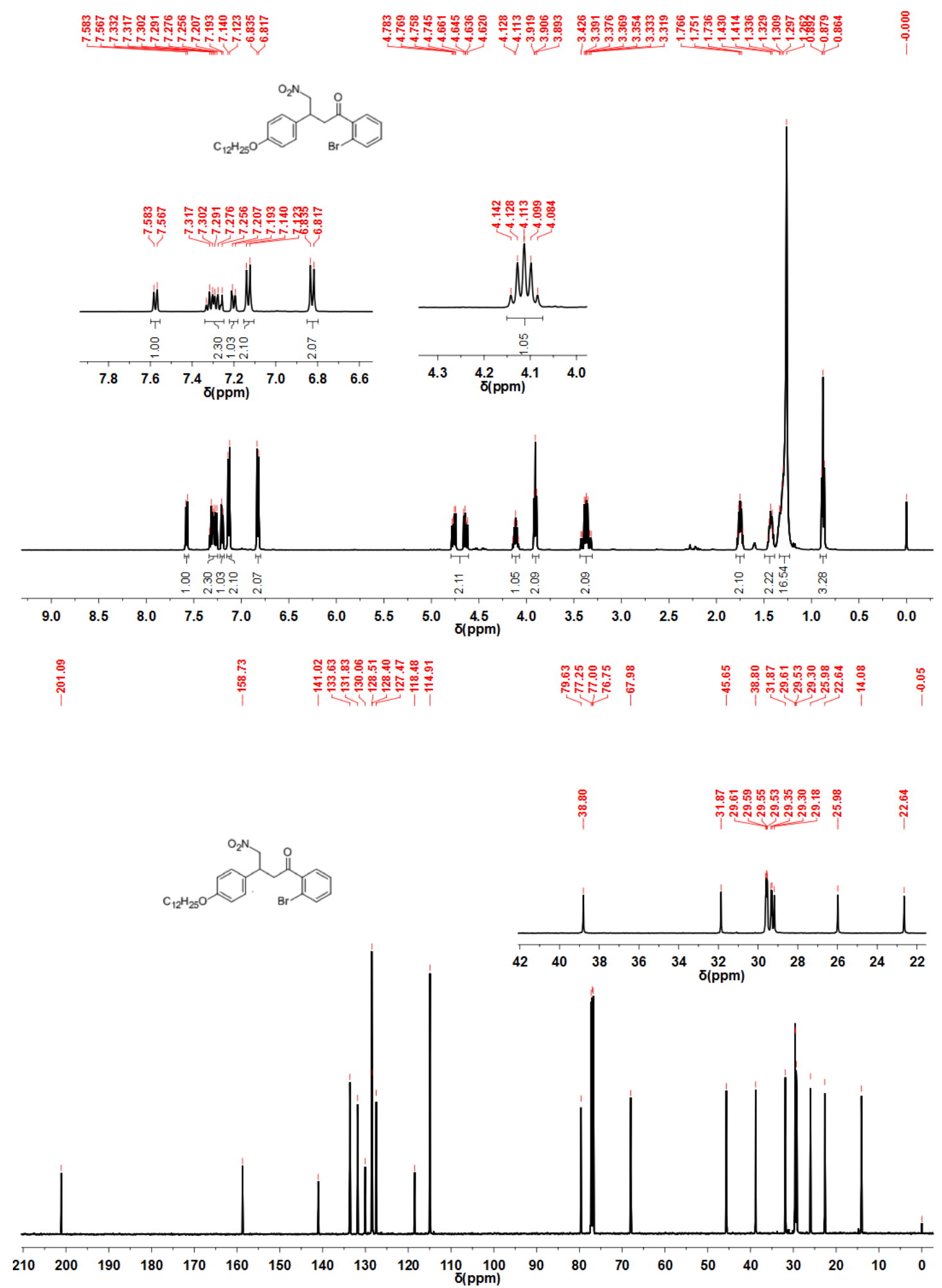
${ }^{1} \mathrm{H}$ NMR and ${ }^{13} \mathrm{C}$ NMR spectrum of $\mathbf{5 c}$ in $\mathrm{CDCl}_{3}$ solution

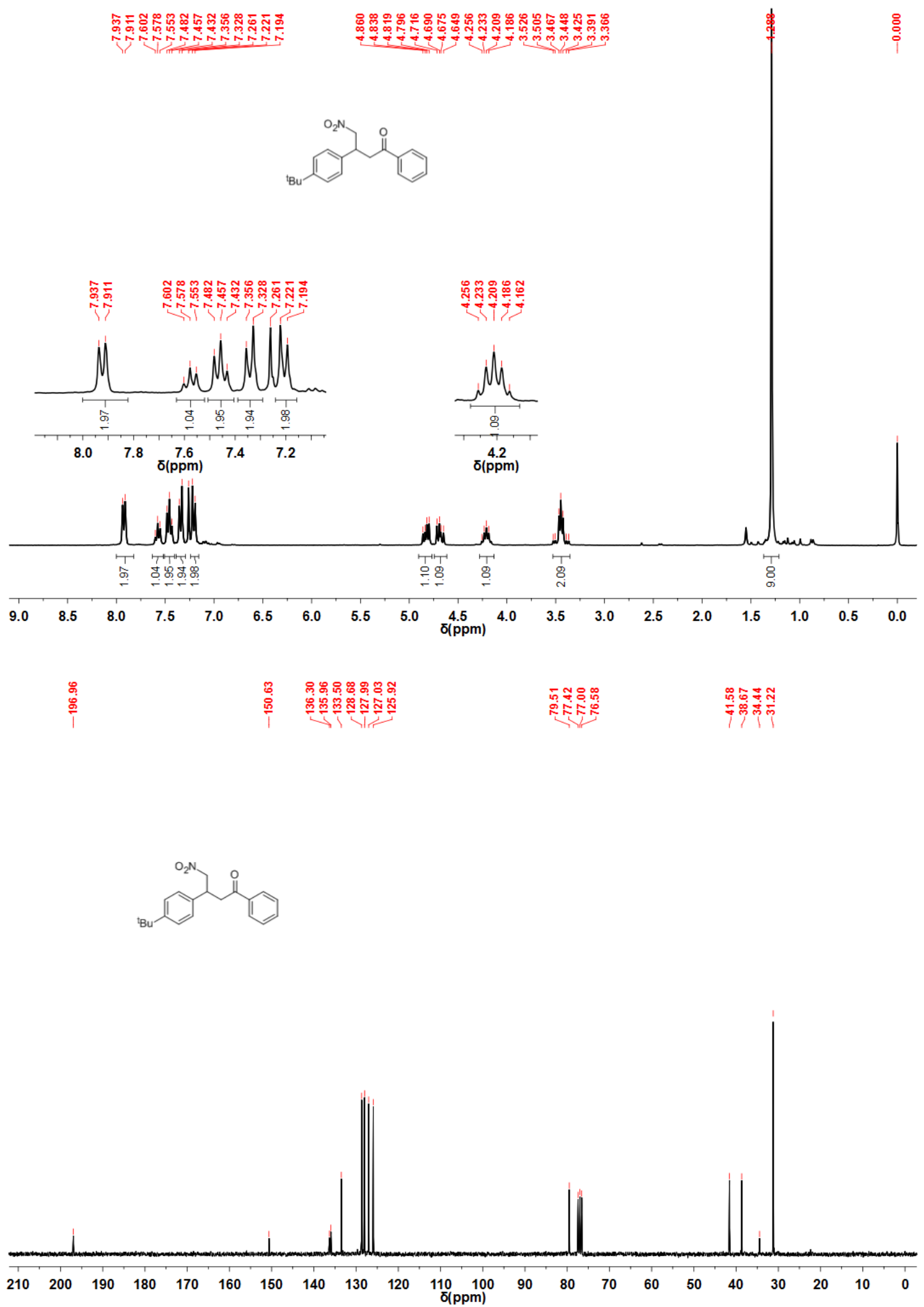


${ }^{1} \mathrm{H}$ NMR and ${ }^{13} \mathrm{C}$ NMR spectrum of $\mathbf{4 a}$ in $\mathrm{CDCl}_{3}$ solution

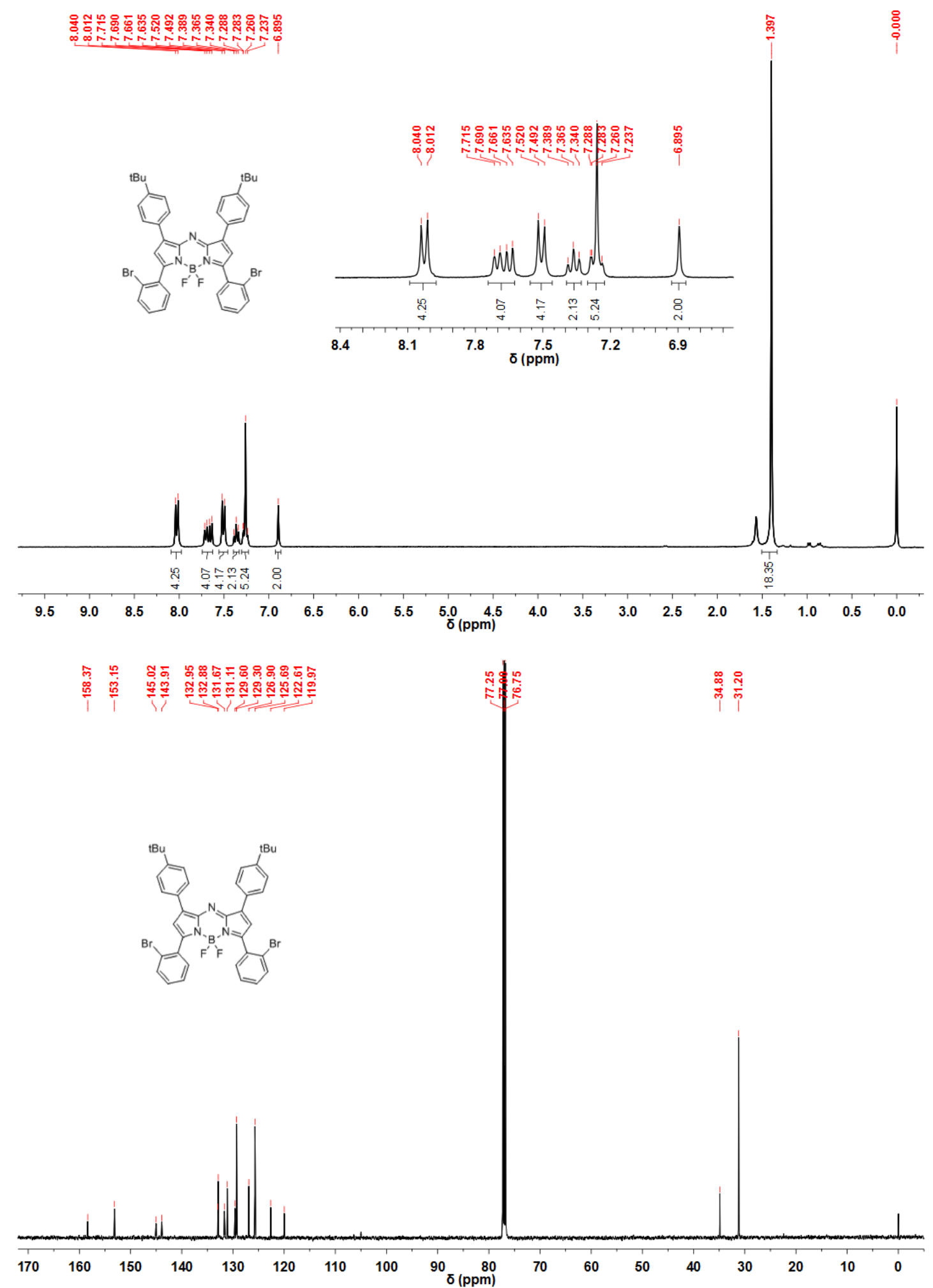


${ }^{1} \mathrm{H}$ NMR and ${ }^{13} \mathrm{C}$ NMR spectrum of $\mathbf{4 b}$ in $\mathrm{CDCl}_{3}$ solution

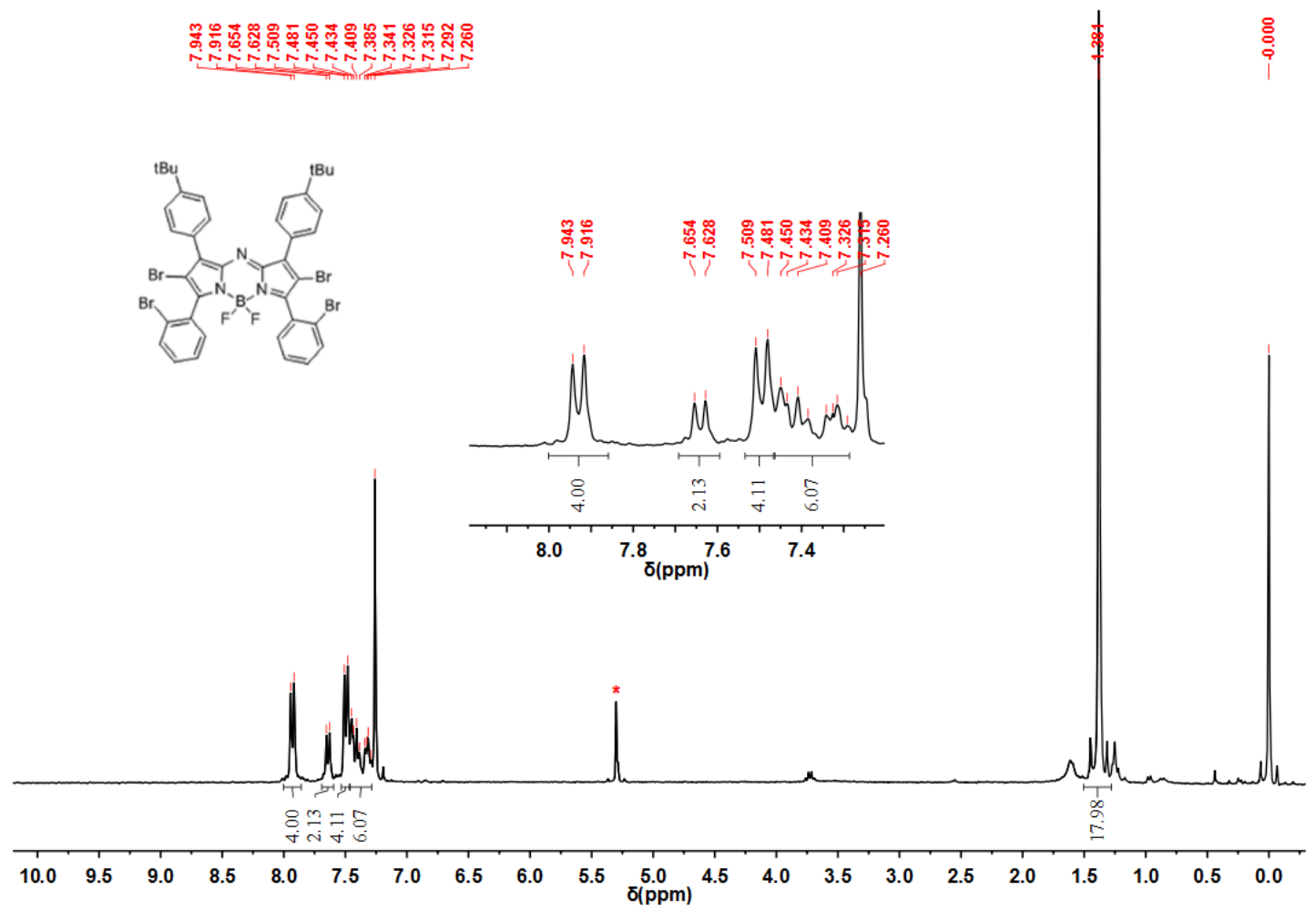

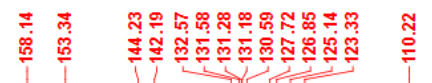
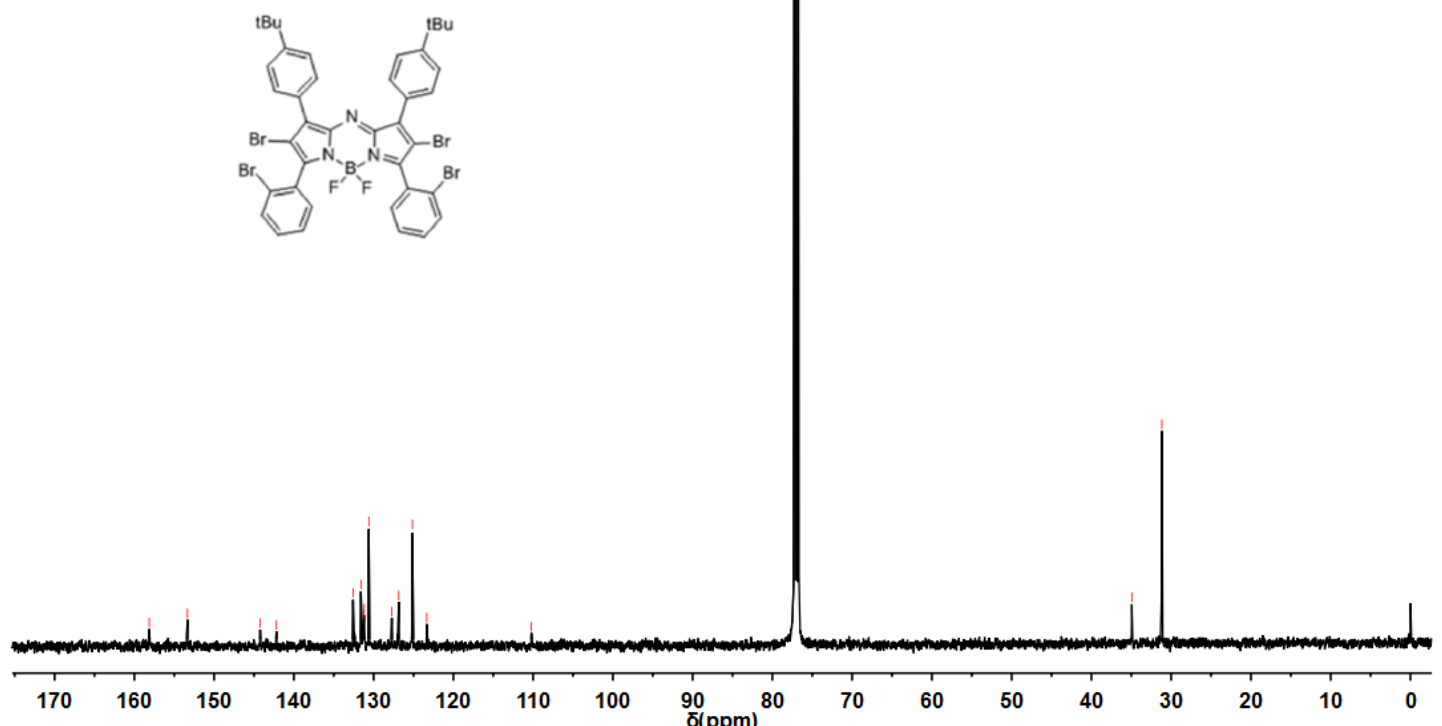
${ }^{1} \mathrm{H}$ NMR and ${ }^{13} \mathrm{C}$ NMR spectrum of $4 \mathbf{c}$ in $\mathrm{CDCl}_{3}$ solution
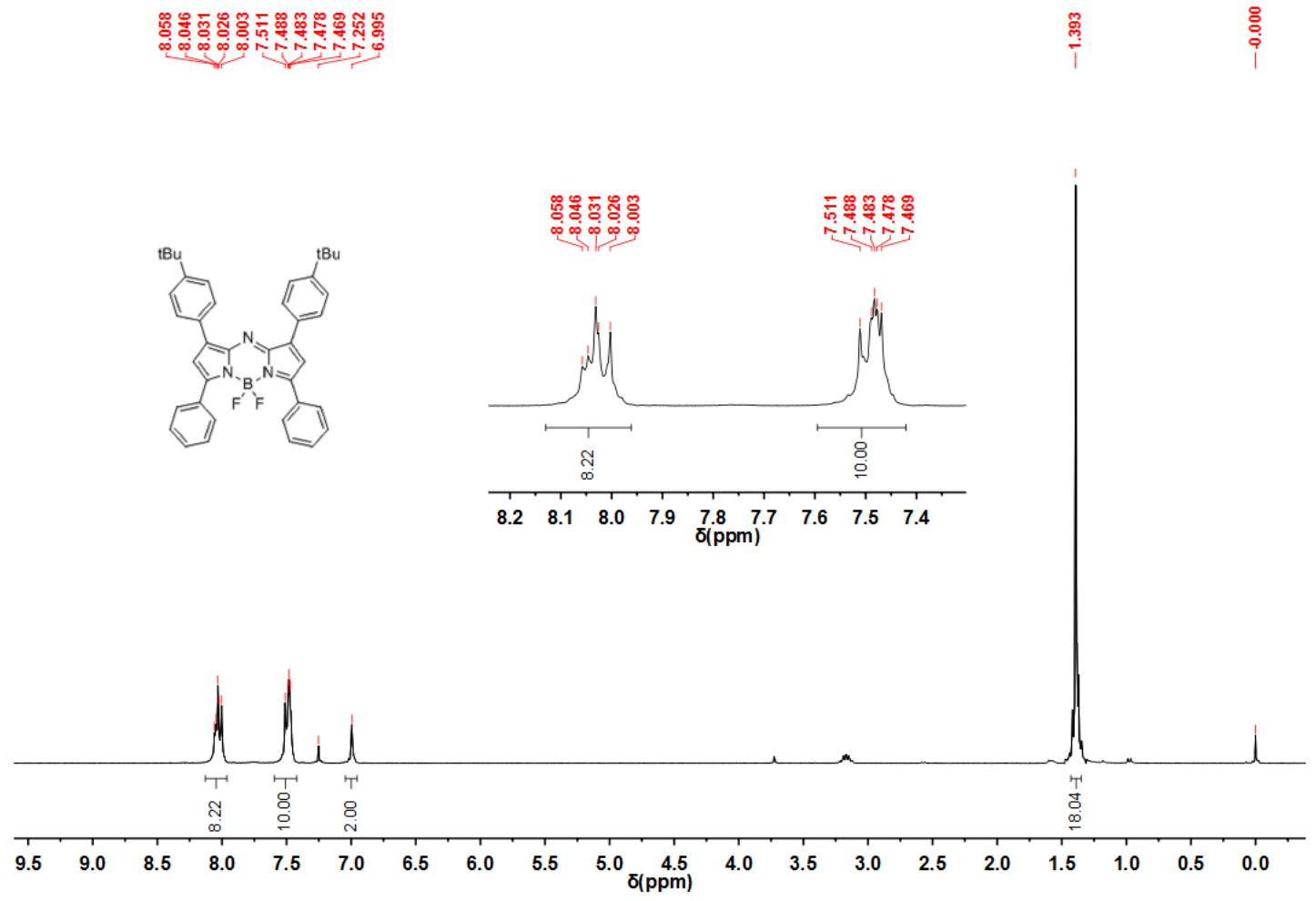

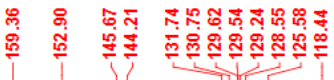

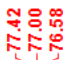

市

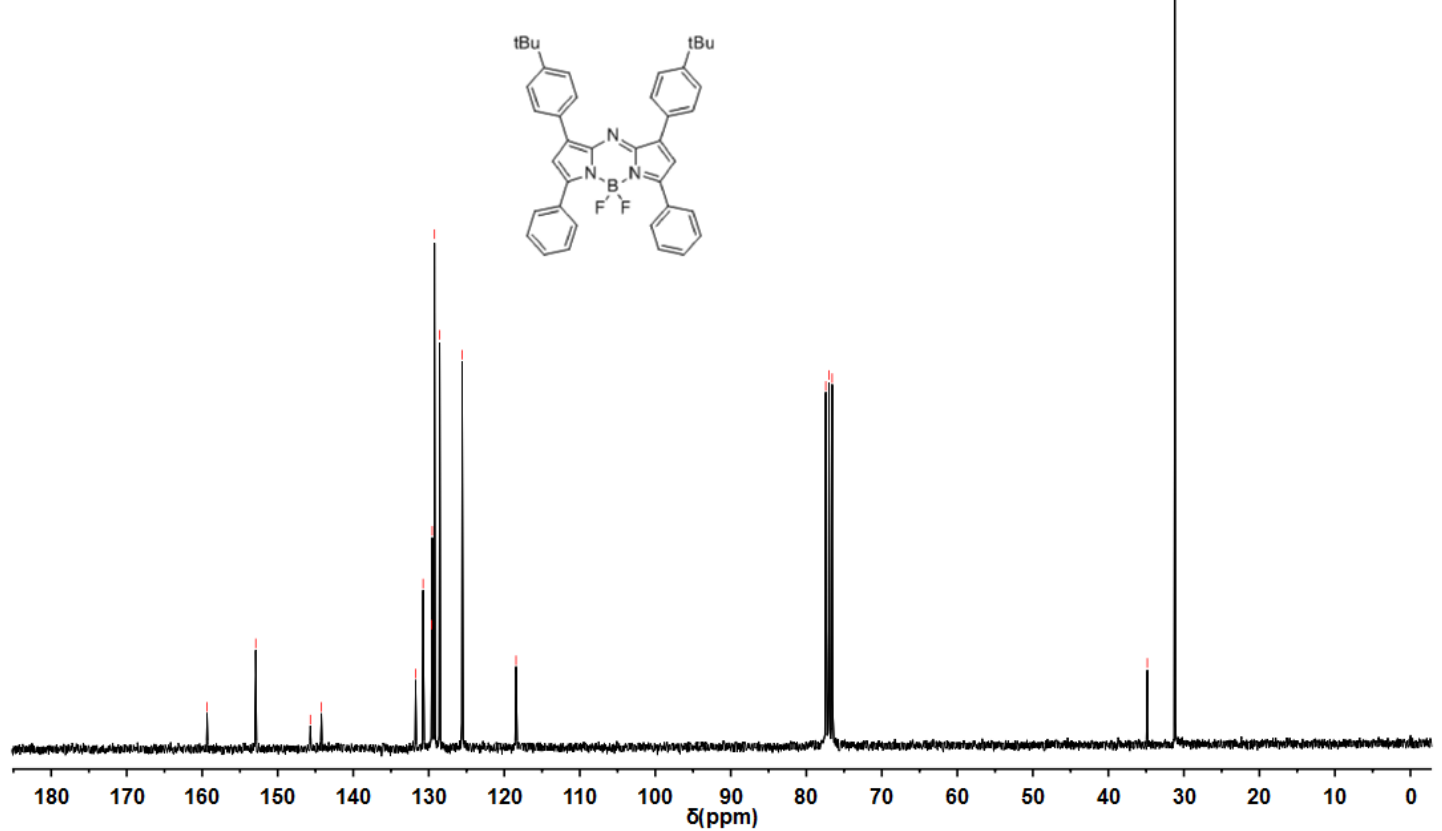


${ }^{1} \mathrm{H}$ NMR and ${ }^{13} \mathrm{C}$ NMR spectrum of $\mathbf{3 a}$ in $\mathrm{CDCl}_{3}$ solution

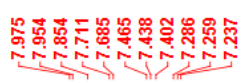
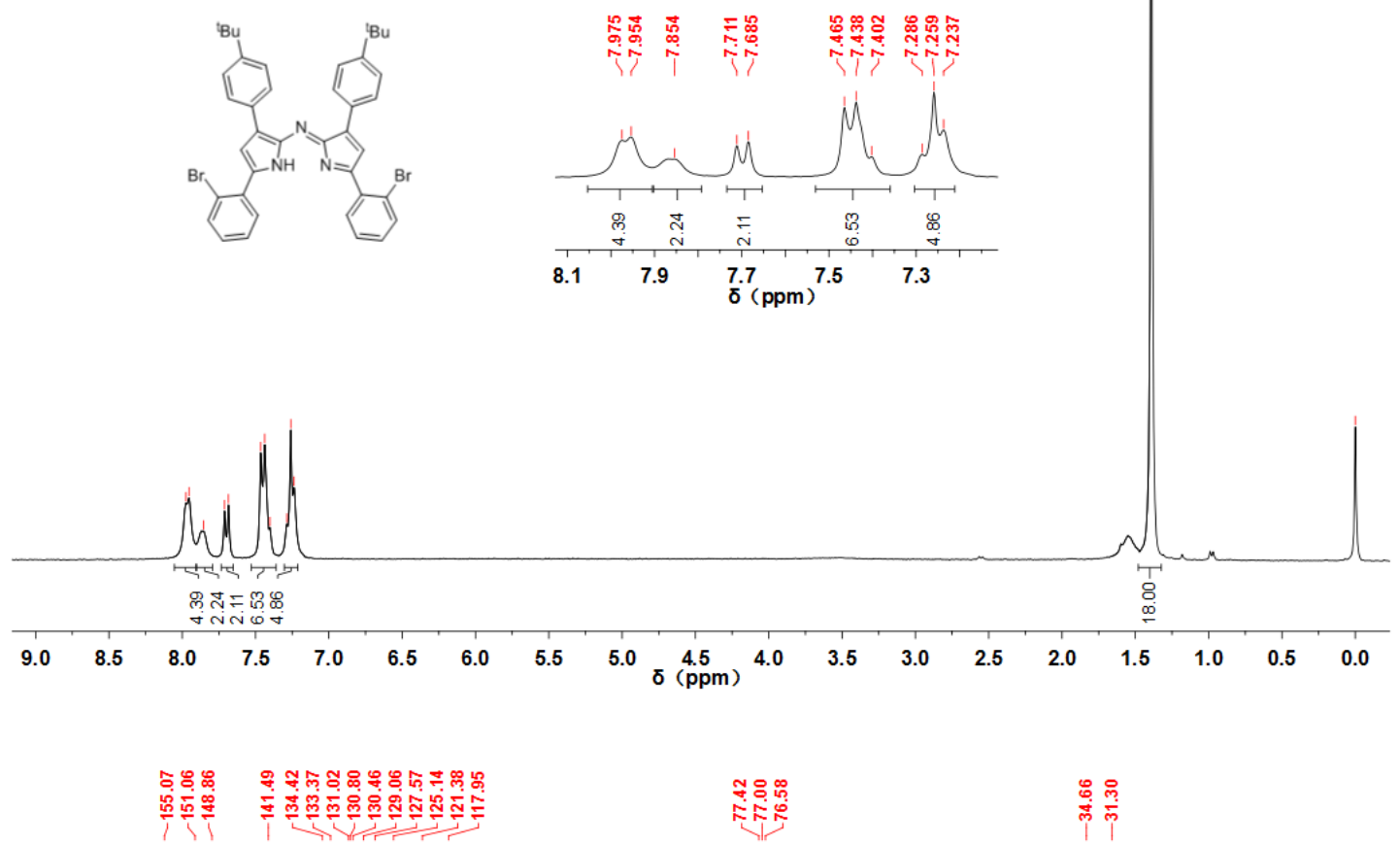

군

芦
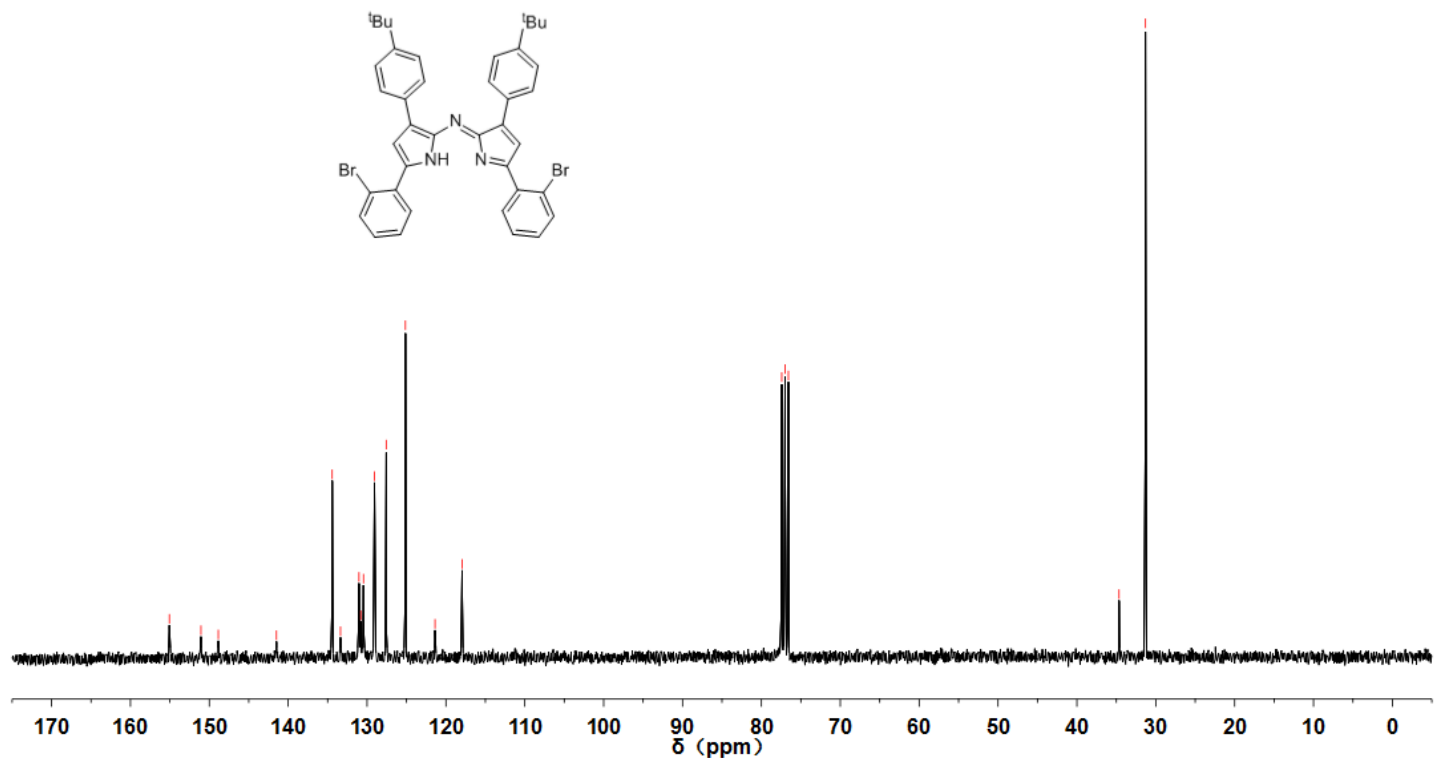
${ }^{1} \mathrm{H}$ NMR and ${ }^{13} \mathrm{C}$ NMR spectrum of $\mathbf{3 b}$ in $\mathrm{CDCl}_{3}$ solution

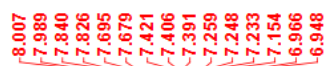

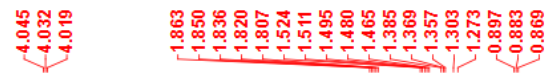

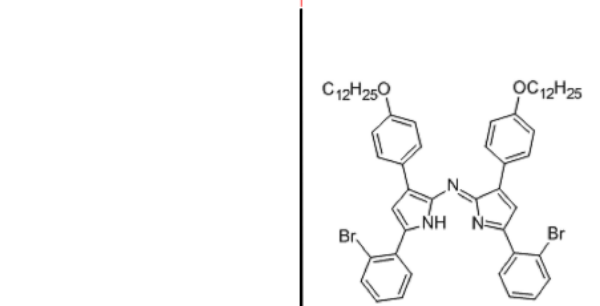

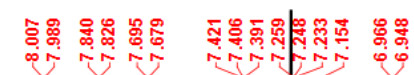

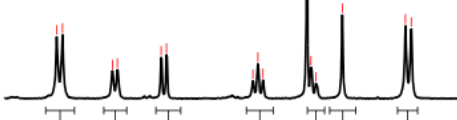

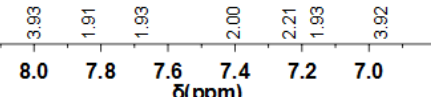

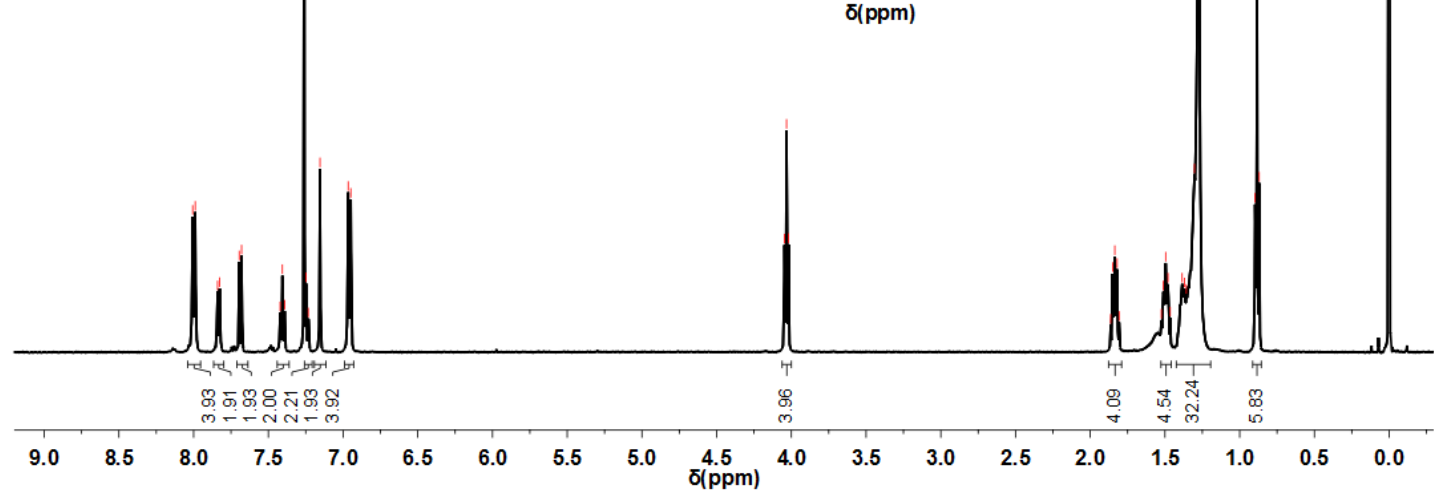

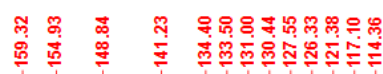

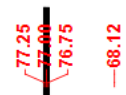

뚜윰유유유유유

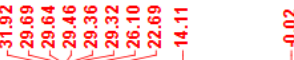
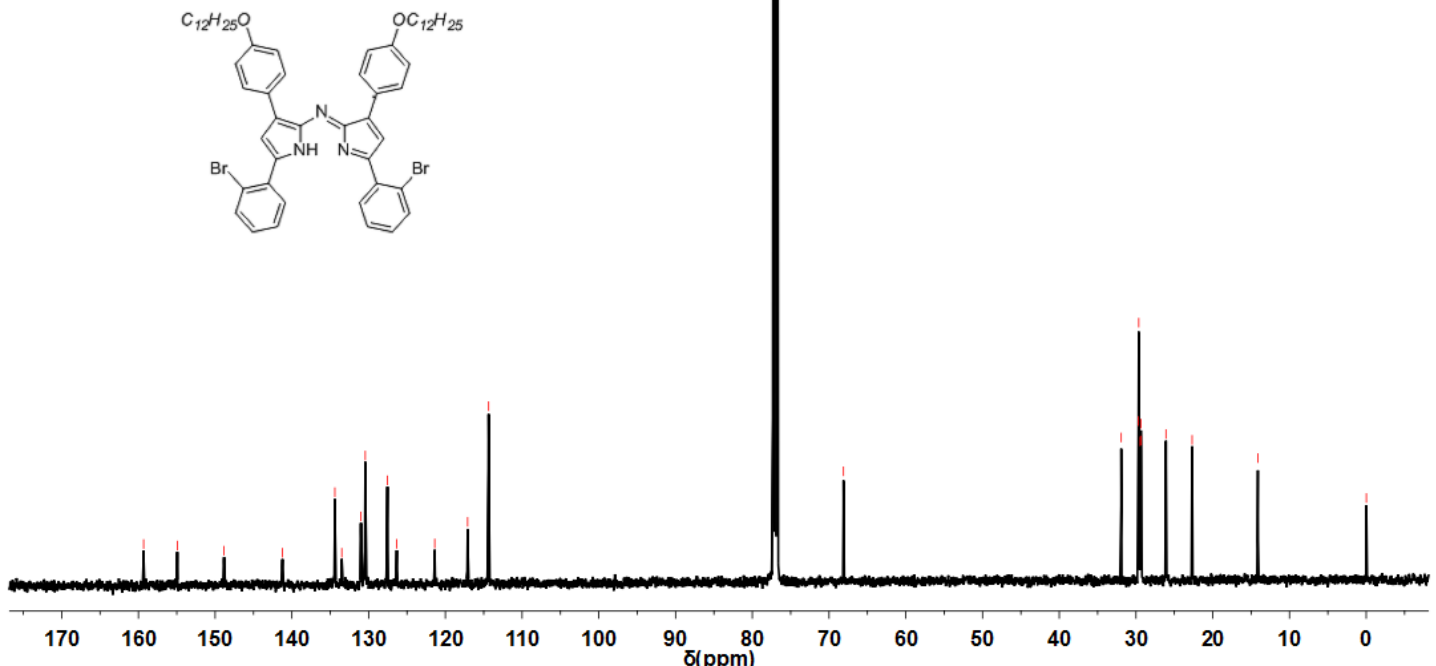
${ }^{1} \mathrm{H}$ NMR and ${ }^{13} \mathrm{C}$ NMR spectrum of $\mathbf{2 a}$ in $\mathrm{CDCl}_{3}$ solution
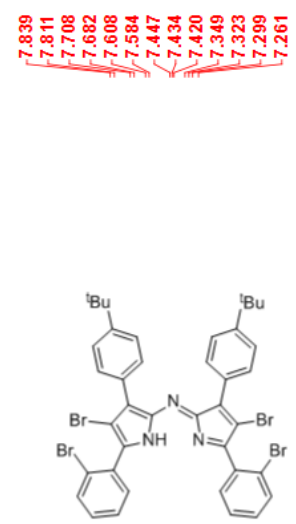

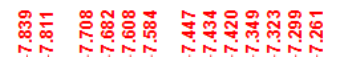
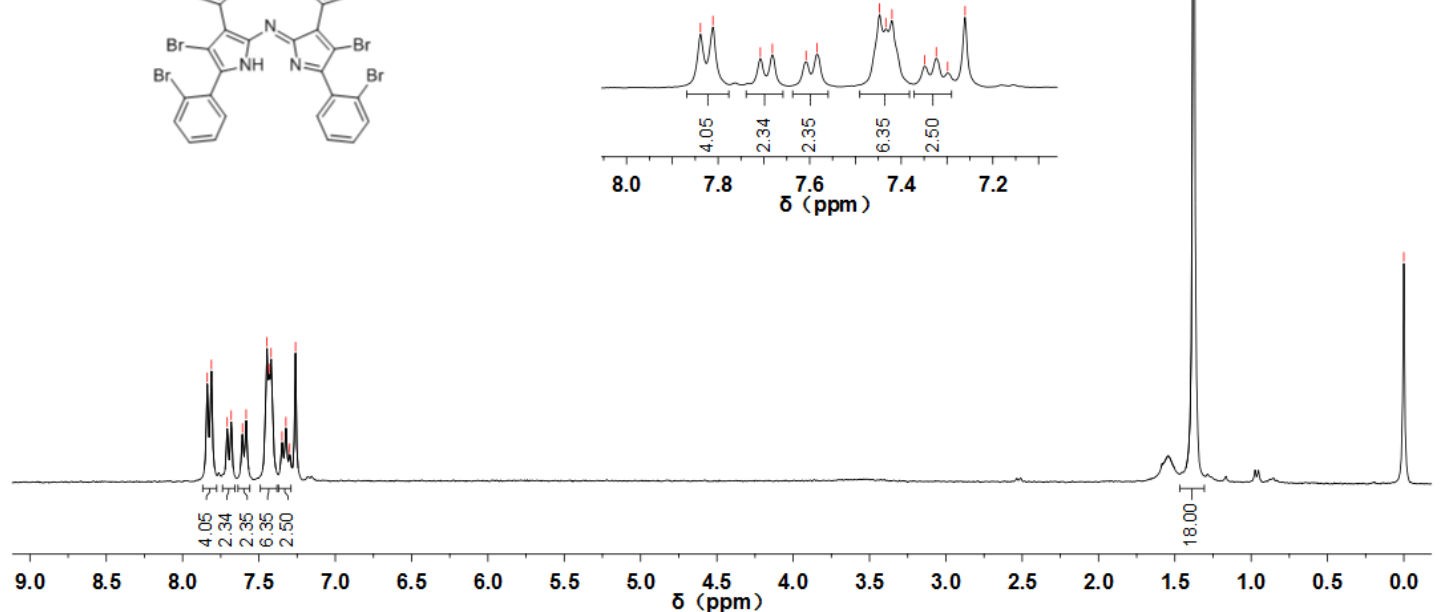

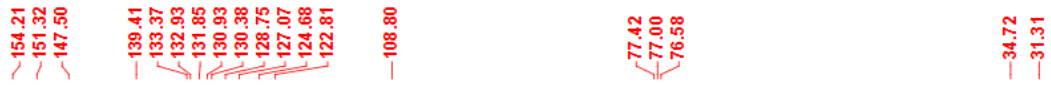
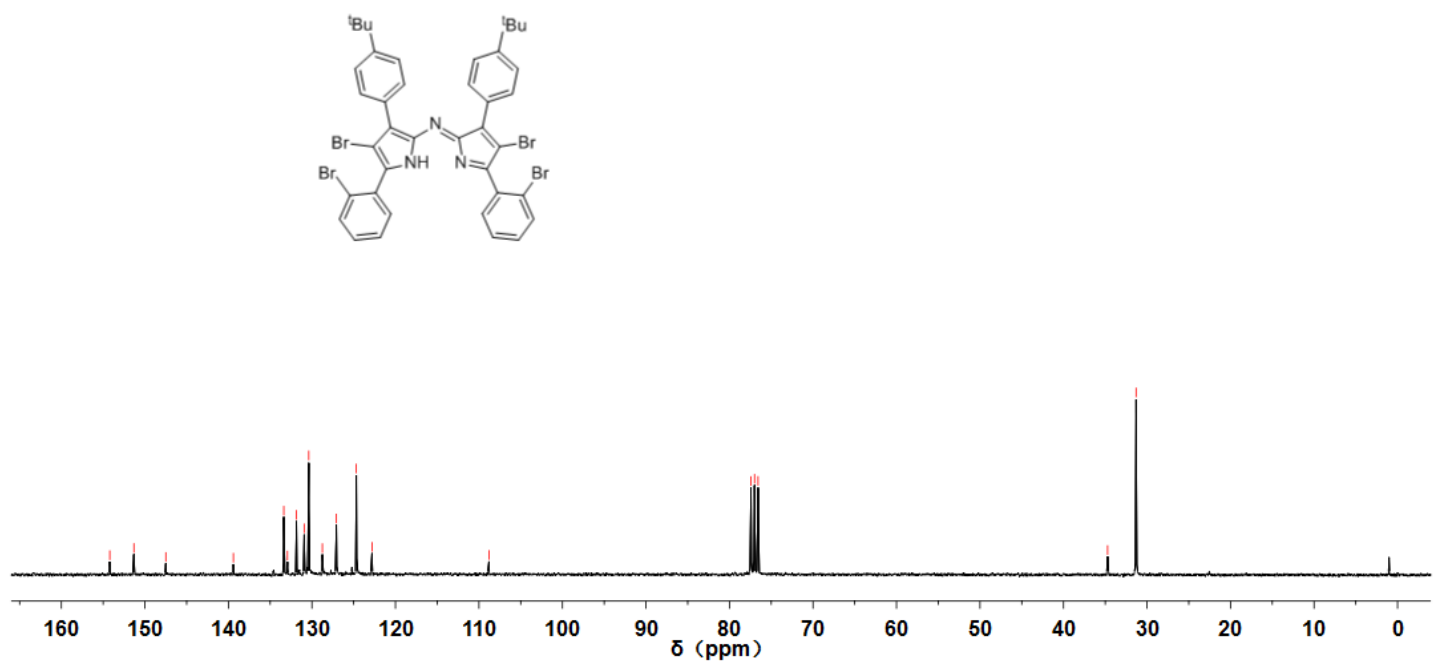
${ }^{1} \mathrm{H}$ NMR and ${ }^{13} \mathrm{C}$ NMR spectrum of $\mathbf{2 b}$ in $\mathrm{CDCl}_{3}$ solution

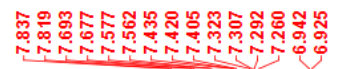

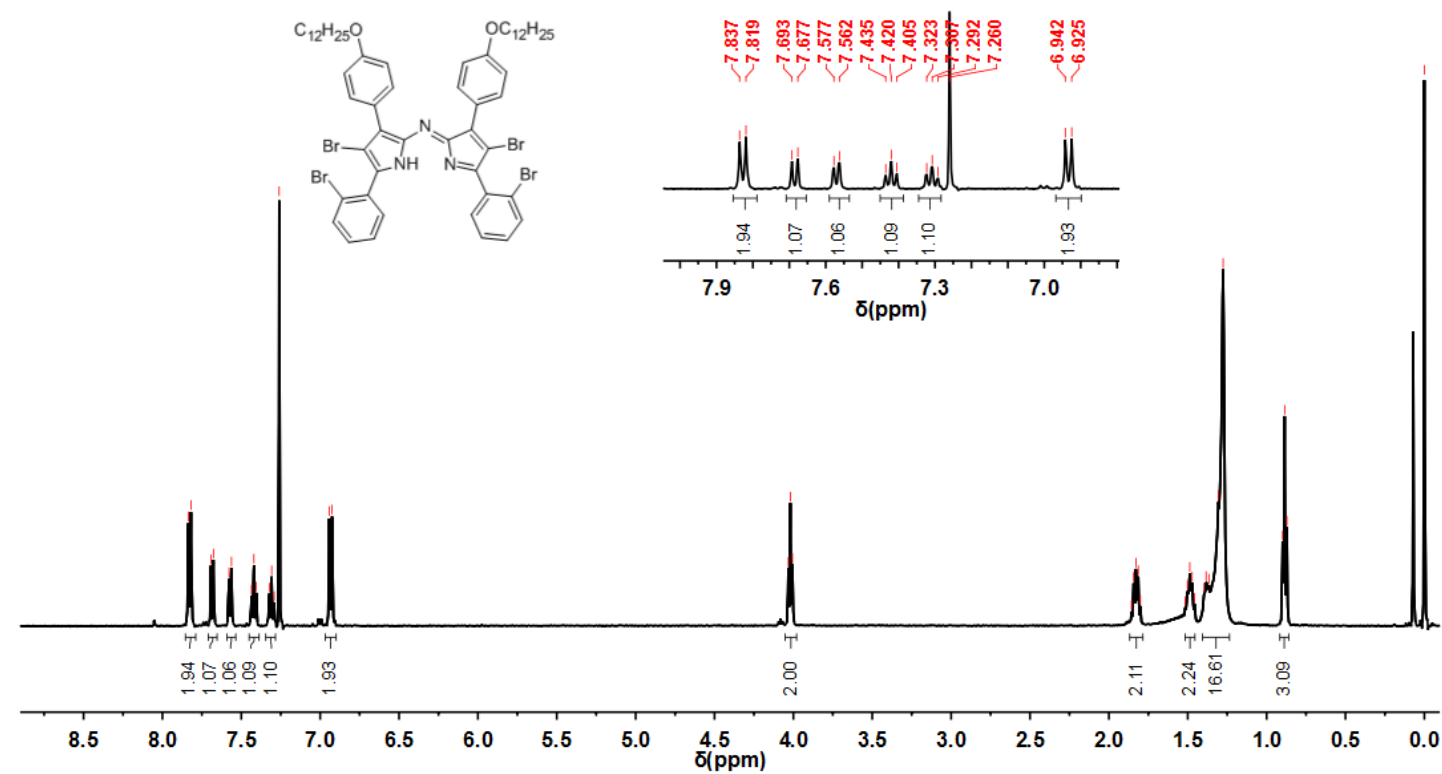

₹
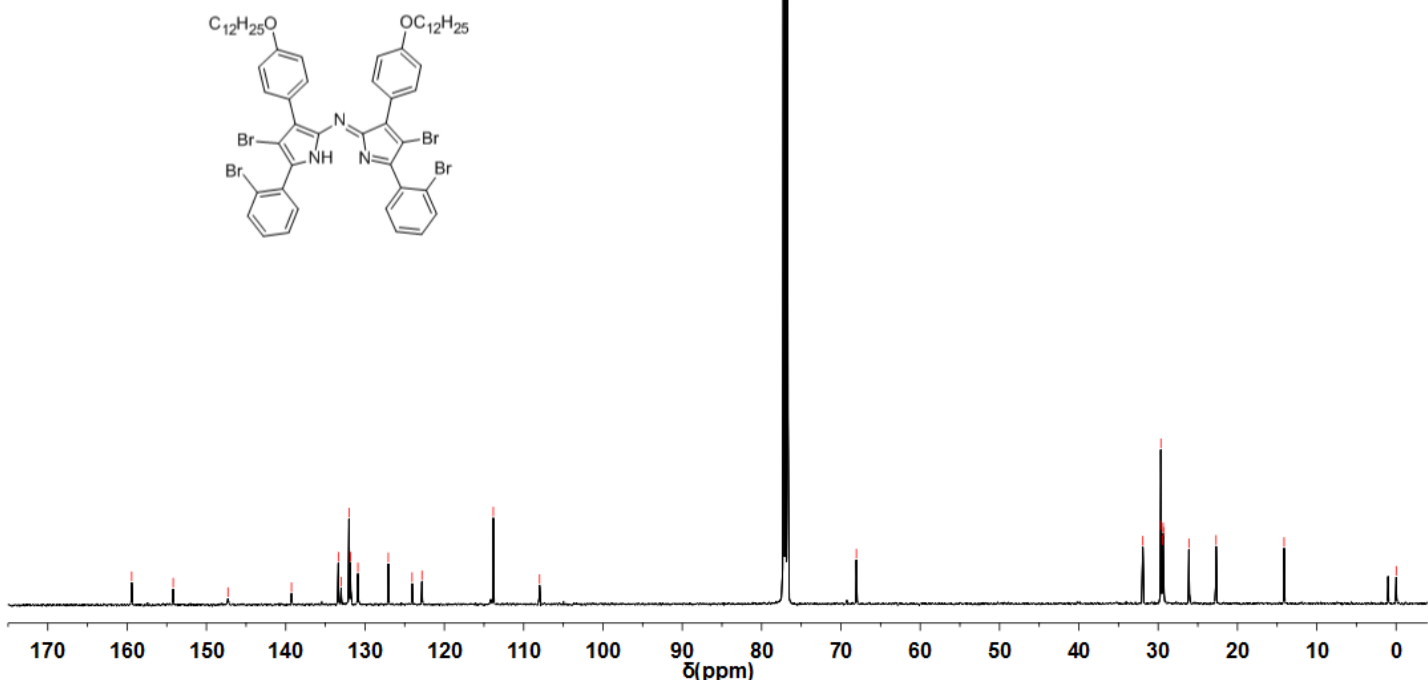
${ }^{1} \mathrm{H}$ NMR and ${ }^{13} \mathrm{C}$ NMR spectrum of $\mathbf{1 a}$ in $\mathrm{CDCl}_{3}$ solution

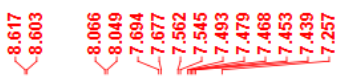
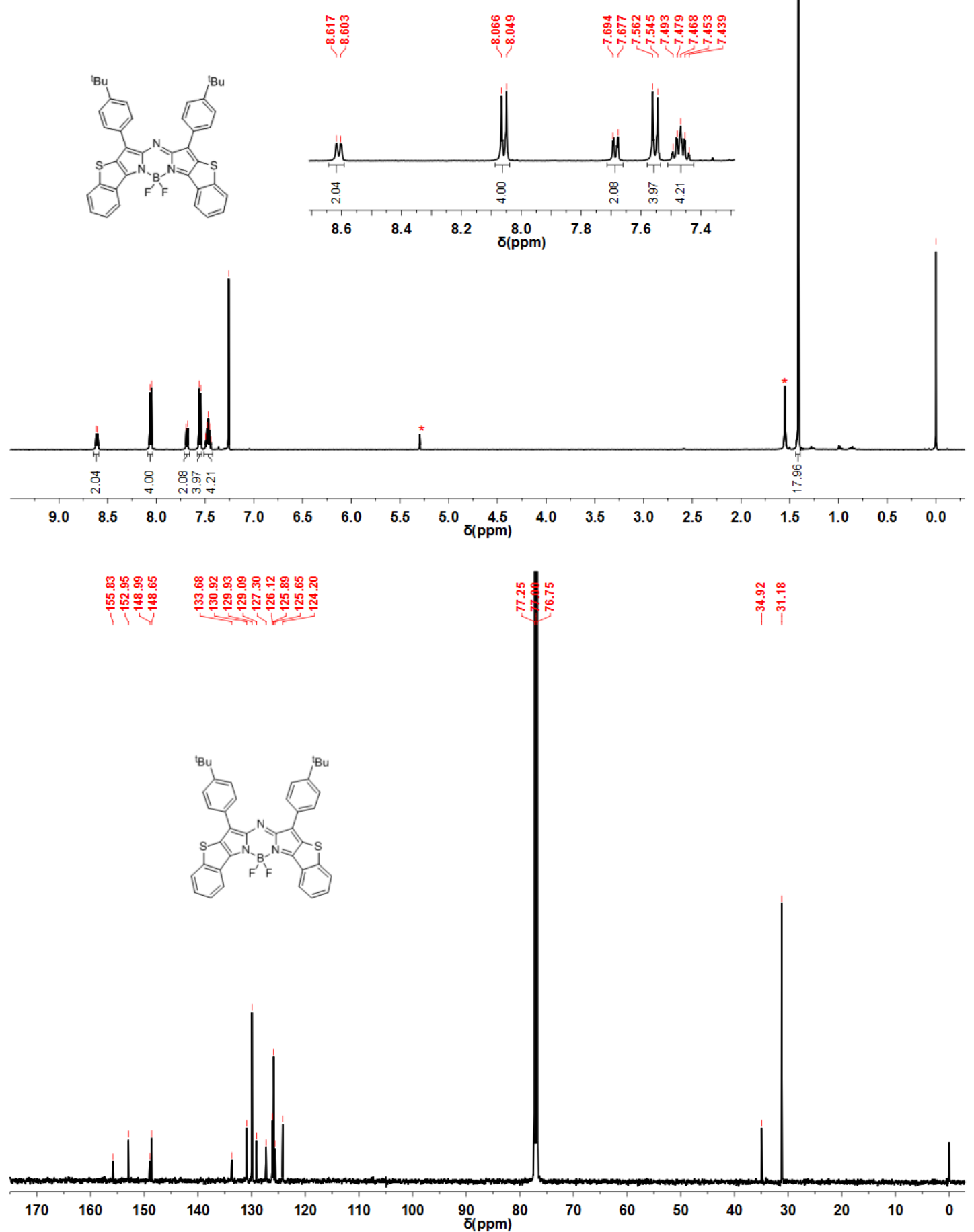
${ }^{1} \mathrm{H}$ NMR and ${ }^{13} \mathrm{C}$ NMR spectrum of $\mathbf{1 b}$ in $\mathrm{CDCl}_{3}$ solution

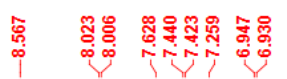
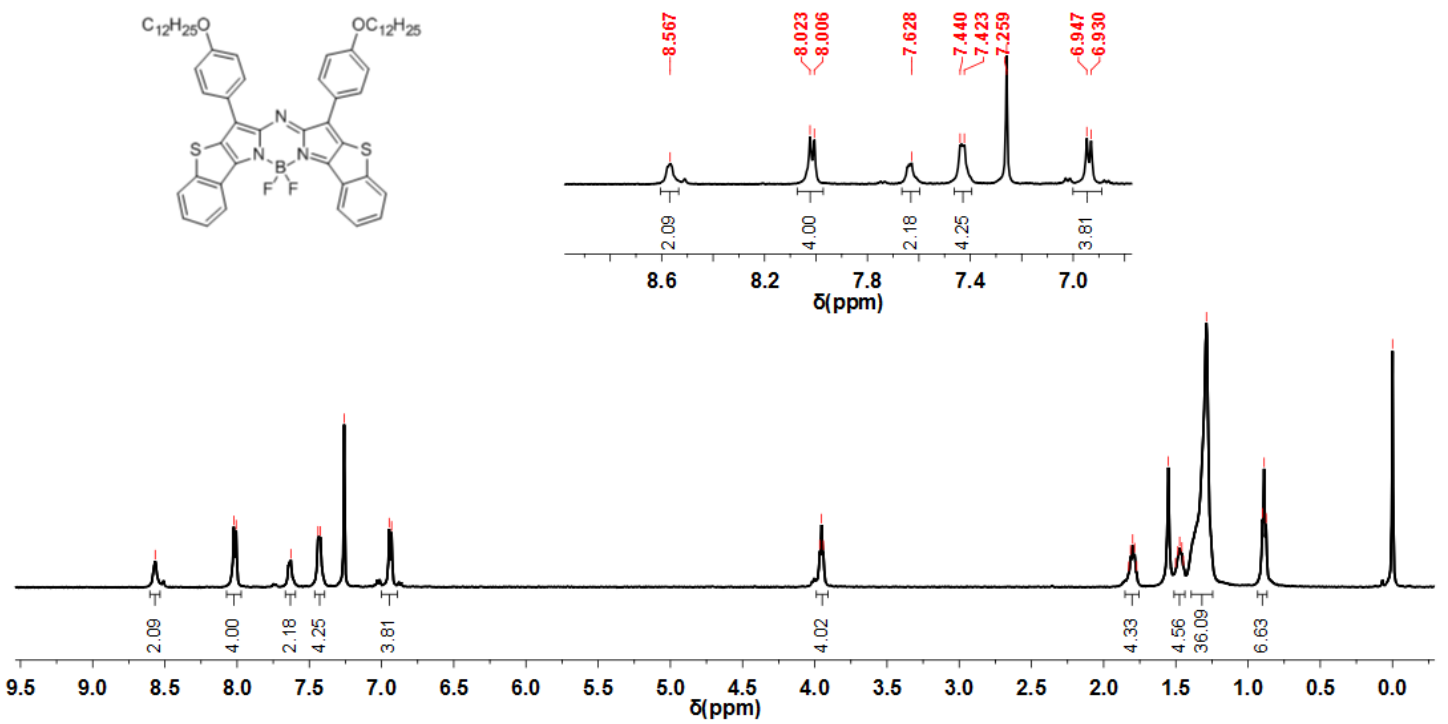

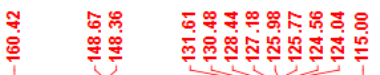

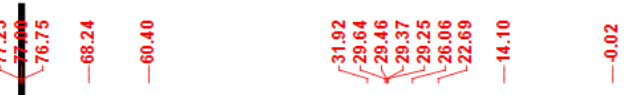
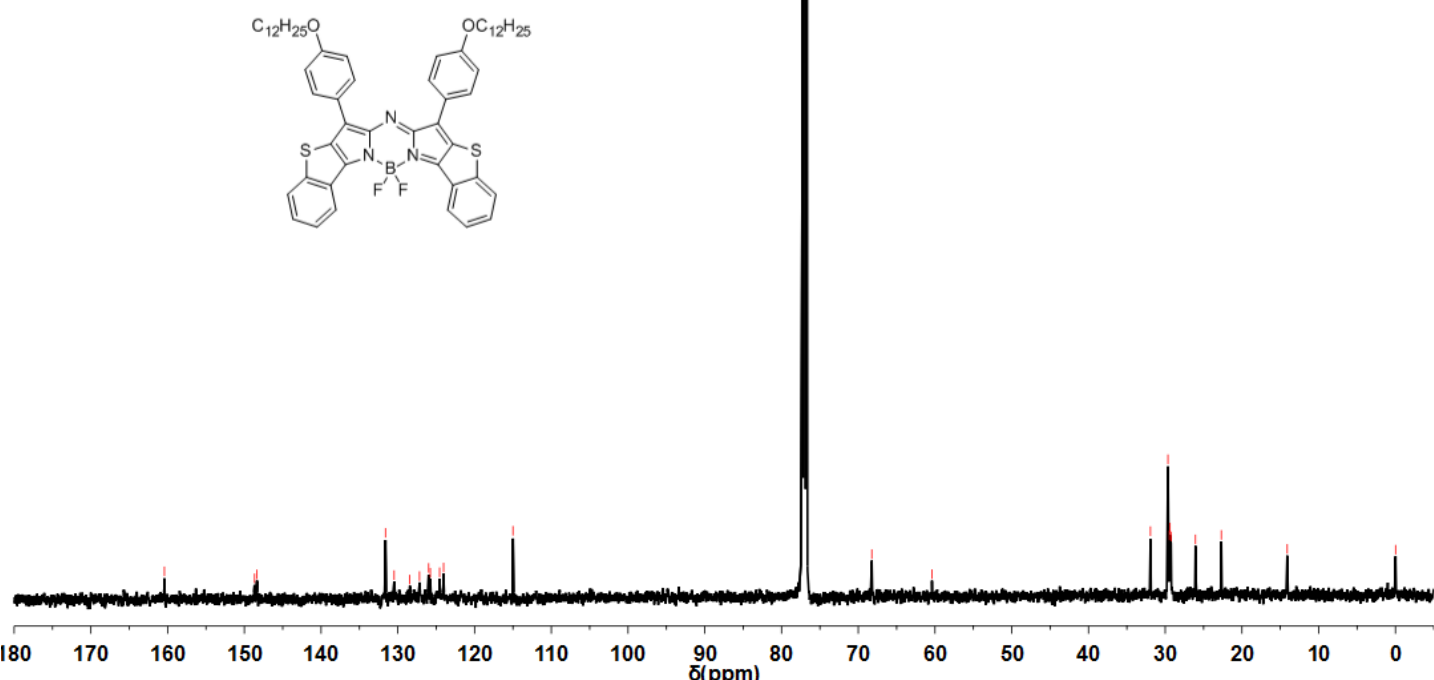
10. High resolution mass spectra for $1 \mathrm{a}$ and $1 \mathrm{~b}$

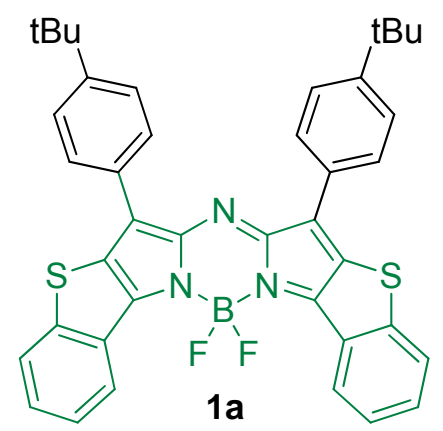

1a HRMS (APCI) calcd. for $\mathrm{C}_{40} \mathrm{H}_{35} \mathrm{BF}_{2} \mathrm{~N}_{3} \mathrm{~S}_{2}[\mathrm{M}+\mathrm{H}]^{+}$: 670.2328, found 670.2332.

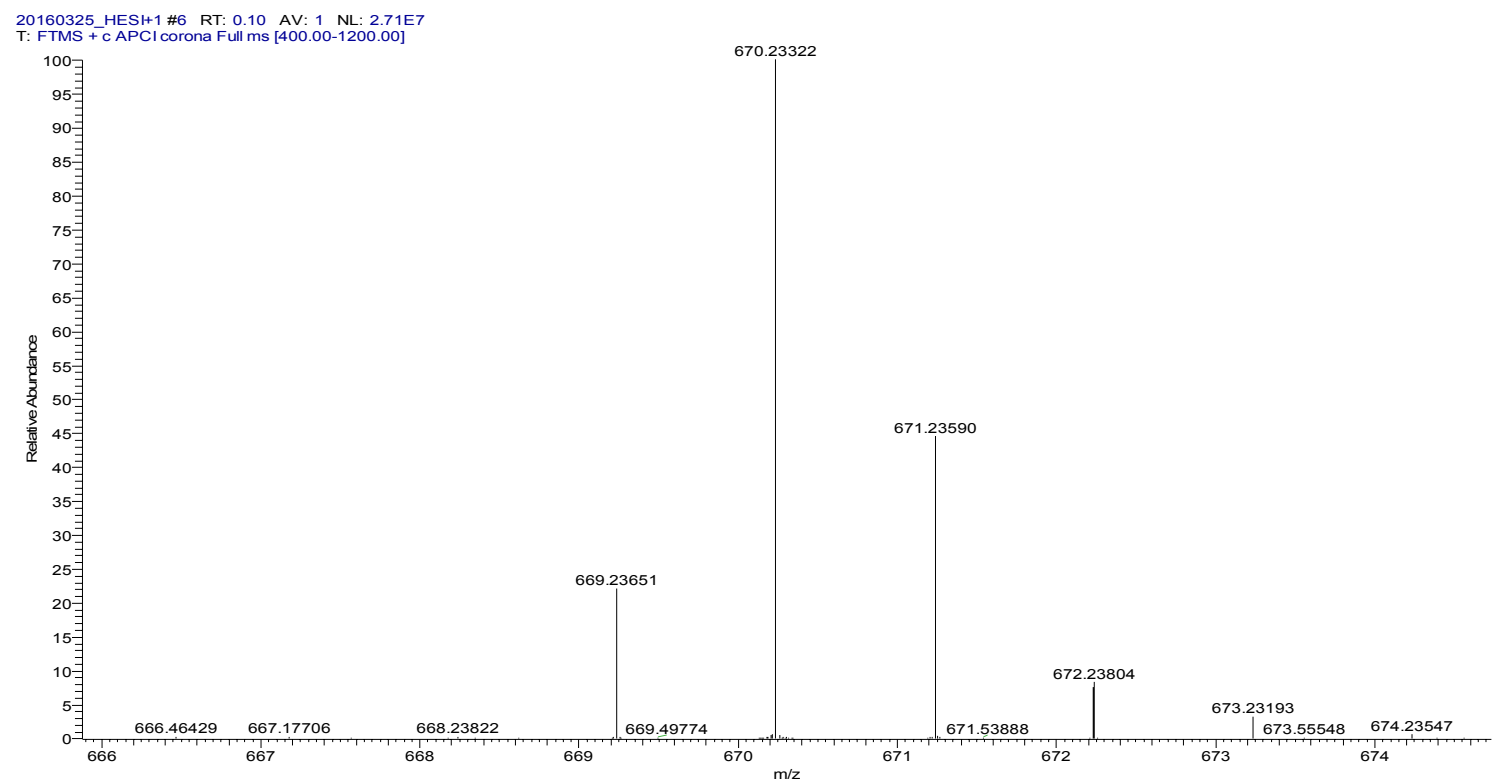




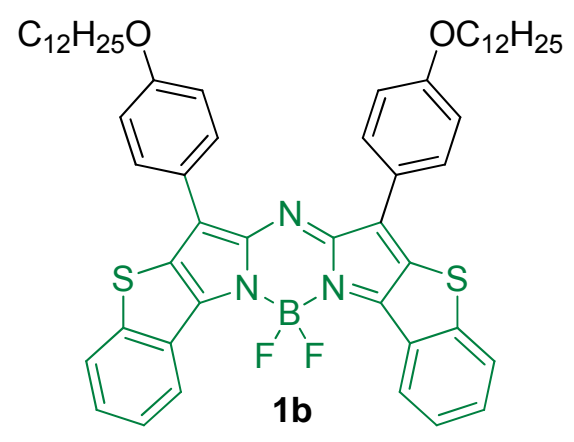

1b HRMS (APCI) calcd. for $\mathrm{C}_{56} \mathrm{H}_{67} \mathrm{BF}_{2} \mathrm{~N}_{3} \mathrm{O}_{2} \mathrm{~S}_{2}[\mathrm{M}+\mathrm{H}]^{+}$: 926.4730 , found 926.4742 .

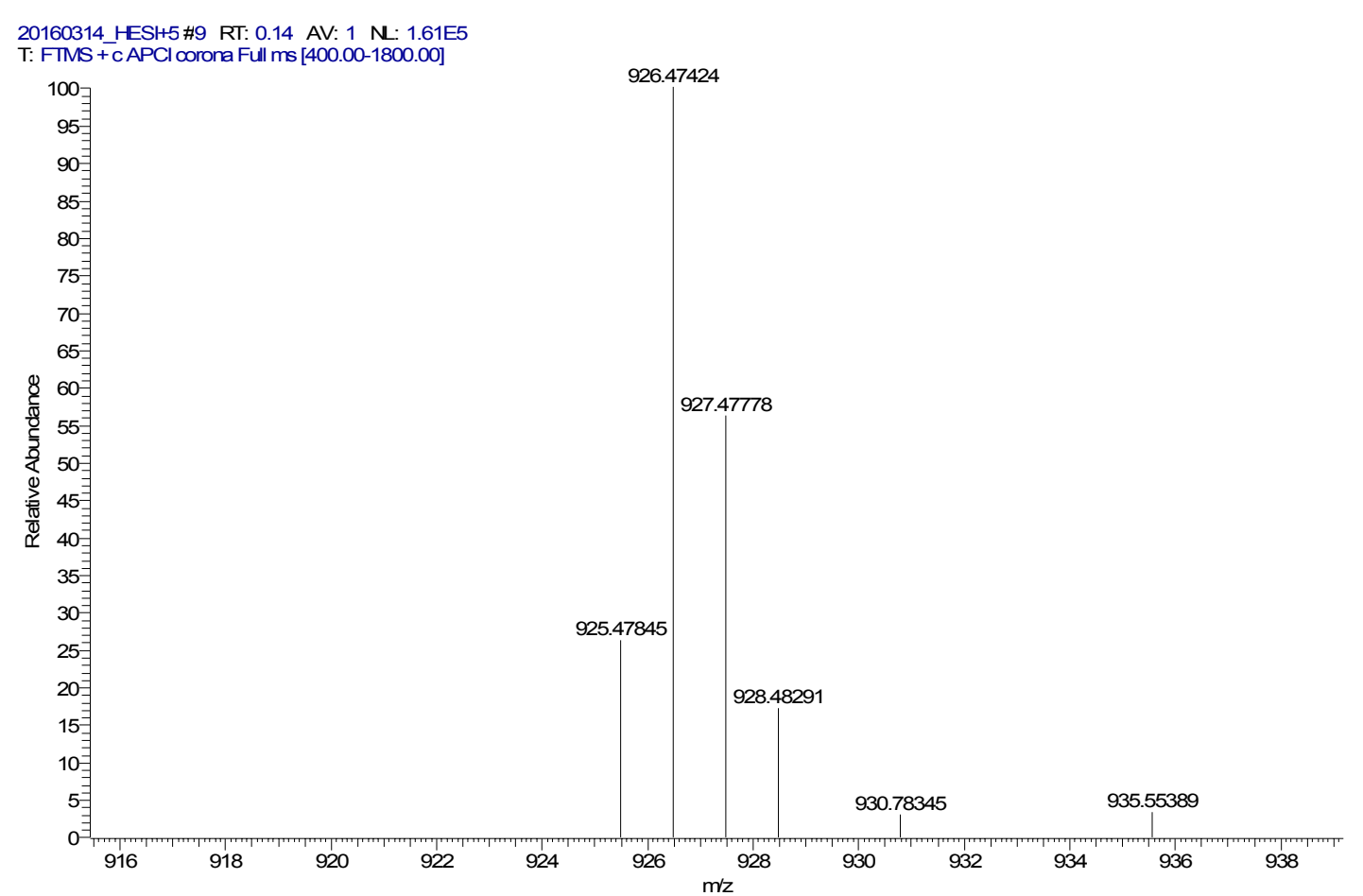




\section{References}

1. Gorman, J. Killoran, C. O' Shea, T. Kenna, W. M. Gallagher and D. F. O'Shea, J. Am. Chem. Soc., 2004, 126, 10619.

2. J. Lakowicz, Principles of Fluorescence Spectroscopy, Springer-Verlag, New York, 3rd edn, 2006.

3. SAINT V 6.01 (NT) Software for the CCD Detector System, Bruker Analytical X-ray Systems, Madison, WI, 1999.

4. G. M. Sheldrick, SHELXS-90, Program for the Solution of Crystal Structure, University of Göttingen, Germany, 1990.

5. SHELXL-97, Program for the Refinement of Crystal Structure, University of Göttingen, Germany, 1997.

6. SHELXTL 5.10 (PC/NT-Version), Program library for StructureSolution and Molecular Graphics, Bruker Analytical X-ray Systems, Madison, WI, 1998.

7. Cui, J.; Sheng, W.; Wu, Q.; Yu, C.; Hao, E.; Bobadova-Parvanova, P.; Storer, M.; Asiri, A. M.; Marwani, H. M.; Jiao, L. Chem. - Asian J. 2017, 12, 2486.

8. Sheng, W.; Zheng, Y.-Q.; Wu, Q.; Wu, Y.; Yu, C.; Jiao, L.; Hao, E.; Wang, J.-Y.; Pei, J. Org. Lett. 2017, 19, 2893.

9. Gaussian 09, Revision D.01, M. J. Frisch, G. W. Trucks, et al., Gaussian, Inc., Wallingford CT, 2013. 